\title{
Estimating local outbreak risks and the effects of non-pharmaceutical interventions in age-structured populations: SARS-CoV-2 as a case study
}

\author{
Francesca A. Lovell-Read ${ }^{\mathrm{a}, *}$, Silvia Shen ${ }^{\mathrm{a}, \mathrm{b}}$, Robin N. Thompson ${ }^{\mathrm{c}, \mathrm{d}}$ \\ ${ }^{a}$ Mathematical Institute, University of Oxford, Oxford, United Kingdom \\ ${ }^{\mathrm{b}}$ Pembroke College, University of Oxford, Oxford, United Kingdom \\ ${ }^{\mathrm{C}}$ Mathematics Institute, University of Warwick, Coventry, United Kingdom \\ ${ }^{\mathrm{d}}$ The Zeeman Institute for Systems Biology and Infectious Disease Epidemiology Research, University of Warwick, Coventry, United Kingdom
}

\section{A R T I C L E I N F O}

\section{Article history:}

Received 5 May 2021

Revised 2 December 2021

Accepted 7 December 2021

Available online 13 December 2021

Dataset link: https://github.com/

francescalovellread/age-dependent-

outbreak-risks

\section{Keywords:}

Mathematical modelling

Infectious disease epidemiology

Outbreak probability

SARS-CoV-2

COVID-19

Age-structured models

Non-pharmaceutical interventions

\begin{abstract}
A B S T R A C T
During the COVID-19 pandemic, non-pharmaceutical interventions (NPIs) including school closures, workplace closures and social distancing policies have been employed worldwide to reduce transmission and prevent local outbreaks. However, transmission and the effectiveness of NPIs depend strongly on age-related factors including heterogeneities in contact patterns and pathophysiology. Here, using SARS-CoV-2 as a case study, we develop a branching process model for assessing the risk that an infectious case arriving in a new location will initiate a local outbreak, accounting for the age distribution of the host population. We show that the risk of a local outbreak depends on the age of the index case, and we explore the effects of NPIs targeting individuals of different ages. Social distancing policies that reduce contacts outside of schools and workplaces and target individuals of all ages are predicted to reduce local outbreak risks substantially, whereas school closures have a more limited impact. In the scenarios considered here, when different NPIs are used in combination the risk of local outbreaks can be eliminated. We also show that heightened surveillance of infectious individuals reduces the level of NPIs required to prevent local outbreaks, particularly if enhanced surveillance of symptomatic cases is combined with efforts to find and isolate nonsymptomatic infected individuals. Our results reflect real-world experience of the COVID-19 pandemic, during which combinations of intense NPIs have reduced transmission and the risk of local outbreaks. The general modelling framework that we present can be used to estimate local outbreak risks during future epidemics of a range of pathogens, accounting fully for age-related factors.
\end{abstract}

(c) 2021 The Authors. Published by Elsevier Ltd. This is an open access article under the CC BY license (http:// creativecommons.org/licenses/by/4.0/).

\section{Introduction}

Throughout the COVID-19 pandemic, policy makers worldwide have relied on non-pharmaceutical interventions (NPIs) to limit the spread of SARS-CoV-2. Commonly introduced NPIs have included school closures, workplace closures and populationwide social distancing policies, all of which aim to reduce the numbers of contacts between individuals and disrupt potential chains of transmission (Perra, 2021; Regmi and Lwin, 2020; Imai et al., 2020; Thompson et al., 2020). Similar measures have previously been adopted for countering other infectious diseases such as Ebola and pandemic influenza (Hatchett et al., 2007; Kirsch et al., 2017; Berkman, 2008), and are likely to remain a key line of defence against emerging pathogens that are directly transmitted

* Corresponding author at: Merton College, Merton Street, Oxford, OX1 4JD, United Kingdom.

E-mail address: francesca.lovell-read@merton.ox.ac.uk (F.A. Lovell-Read). between hosts. NPIs are particularly important when no effective treatment or vaccine is available, and they are also beneficial when vaccination programmes are being rolled out (Moore et al., 2021; Spinelli et al., 2021; Thompson et al., 2021). If vaccines do not prevent transmission completely, then NPIs may be important even when vaccination is widespread (Sachak-Patwa et al., 2021). However, the negative economic, social and non-disease health consequences of NPIs have been widely discussed, with the impact of school closures on the academic progress and wellbeing of school-aged individuals a particular concern (Berkman, 2008; Donohue and Miller, 2020; Silverman et al., 2020; Sadique et al., 2008; Brooks et al., 2020; Loades et al., 2020). Therefore, assessing the effectiveness of different NPIs at reducing transmission is critical for determining whether or not they should be used.

Since NPIs such as school and workplace closures affect distinct age groups within the population, when evaluating their effectiveness it is important to account for age-dependent factors that 
influence transmission. Multiple studies have documented marked heterogeneities in the patterns of contacts between individuals in different age groups, with school-aged individuals tending to have more contacts each day than older individuals (Prem et al., 2017; Mossong et al., 2008; Danon et al., 2013; Leung et al., 2017; Béraud et al., 2015; Ibuka et al., 2016; Read et al., 2014). Since close contact between individuals is a key driver of transmission for respiratory pathogens such as influenza viruses and SARS-CoV-2, these contact patterns influence transmission dynamics and consequently the effects of interventions that target different age groups (Mossong et al., 2008; Danon et al., 2013; Read et al., 2012; Wallinga et al., 2006; Kucharski et al., 2014; Zhang et al., 2020). Additionally, many diseases are characterised by significant agerelated variations in pathophysiology. For example, for SARSCoV-2, children may be less susceptible to infection than adults (Zhang et al., 2020; Munro and Faust, 2020; Viner et al., 2021; Goldstein et al., 2020; Davies et al., 2020b), and more likely to experience asymptomatic or subclinical courses of infection (Munro and Faust, 2020; Davies et al., 2020b; Ludvigsson, 2020; Dong et al., 2020; Siebach et al., 2021; Mehta et al., 2020; Han et al., 2021). Since the secondary attack rate (the proportion of close contacts that lead to new infections) from asymptomatic or subclinical hosts is lower than from hosts with clinical symptoms (Pollock and Lancaster, 2020; Buitrago-Garcia et al., 2020; Byambasuren et al., 2020; Koh et al., 2020; Madewell et al., 2020; Qiu et al., 2021), children are likely to be less infectious on average than older individuals who are at increased risk of developing symptoms (Pijls et al., 2021; Verity et al., 2020; Chen et al., 2020).

Previous studies have used age-stratified deterministic transmission models to investigate the effects of NPIs on COVID-19 epidemic peak incidence and timing. Prem et al. (2020) projected the outbreak in Wuhan, China, over a one year period under different control scenarios, and demonstrated that a period of intense control measures including school closures, a 90\% reduction in the workforce and a significant reduction in other social mixing could delay the epidemic peak by several months. Zhang et al. (2020) predicted that eliminating all school contacts during the outbreak period would lead to a noticeable decrease in the peak incidence and a later peak; however, they did not take differences between symptomatic and asymptomatic cases into account explicitly. In contrast, Davies et al. (2020b) used estimates of age-dependent susceptibility and clinical fraction fitted to the observed age distribution of cases in six countries to demonstrate that school closures alone were unlikely to reduce SARS-CoV-2 transmission substantially. Davies et al. (2020a) subsequently concluded that a combination of several strongly enforced NPIs would be necessary to avoid COVID-19 cases exceeding available healthcare capacity in the UK.

Rather than considering the entire epidemic curve, here we focus on estimating the probability that cases introduced to a new location trigger a local outbreak as opposed to fading out with few cases. Localised clusters of transmission have been a feature of the COVID-19 pandemic (Liu et al., 2020; Leclerc et al., 2020; Rossman et al., 2020), and assessing the risk that such local outbreaks occur requires a stochastic model in which the pathogen can either invade or fade out. Stochastic branching process models have been applied previously to assess outbreak risks for many pathogens without considering different age groups explicitly (Althaus et al., 2015; Thompson et al., 2019; Thompson, 2020; Hellewell et al., 2020; Lovell-Read et al., 2021), and extended to consider adults and children as two distinct groups (Nishiura et al., 2011). However, the significant heterogeneities in contact patterns and pathophysiology between individuals across the full range of ages have never previously been considered in estimates of local outbreak risks. Here, we develop an age-structured branch- ing process model that can be used to estimate the probability of a local outbreak occurring for index cases of different ages, and demonstrate how the age-dependent risk profile changes when susceptibility to infection and clinical fraction vary with age.

We use the model to investigate the effects on the local outbreak probability of NPIs that reduce the numbers of contacts between individuals. Specifically, we use location-specific contact data for the UK detailing the average numbers of daily contacts occurring in school, in the workplace and elsewhere (Prem et al., 2017) to model the impacts of school closures, workplace closures and broader social distancing policies. We demonstrate that, for SARS-CoV-2, contacts occurring outside schools and the workplace are a key driver of sustained transmission. Thus, population-wide social distancing policies that affect individuals of all ages lead to a substantial reduction in the risk of local outbreaks. In contrast, since school-aged individuals only make up around one quarter of the UK population and tend to have large numbers of contacts outside school, school closures are predicted to have only a limited effect when applied as the sole NPI.

We then go on to consider the impacts of mixed strategies made up of multiple NPIs, as well as additional NPIs that do not only reduce numbers of contacts. Specifically, we show that rigorous surveillance and effective isolation of infected hosts can reduce the level of contact-reducing NPIs required to achieve substantial reductions in the risk of local outbreaks. Although we use SARS$\mathrm{CoV}-2$ as a case study, our approach can be applied more generally to explore the effects of NPIs on the risk of outbreaks of any pathogen for which age-related heterogeneities play a significant role in transmission dynamics.

\section{Methods}

\subsection{Mathematical model}

We considered a branching process model in which the population was divided into 16 age groups, denoted $G_{1}, G_{2}, \cdots, G_{16}$. The first 15 groups represent individuals aged $0-74$, divided into fiveyear intervals $(0-4,5-9,10-14$ etc.). The final group represents individuals aged 75 and over. The total number of individuals in age group $G_{k}$ is denoted $N_{k}$. Infected individuals in each age group $G_{k}$ are classified into compartments representing asymptomatic $\left(A_{k}\right)$, presymptomatic $\left(P_{k}\right)$ or symptomatic $\left(S_{k}\right)$ hosts, where an individual in the $A_{k}$ compartment does not develop symptoms at any time during their course of infection.

An infected individual of any type in group $G_{k}$ may generate new infections in any age group. In our model, the rate at which a single infected symptomatic individual in group $G_{k}$ generates infections in group $G_{j}$ is given by

$\beta_{k j}=B \tau_{k} \omega_{j} C_{k j}$.

Here, $\tau_{k}$ represents the infectivity of individuals in group $G_{k}, \omega_{j}$ represents the susceptibility to infection of individuals in group $G_{j}$, $C_{k j}$ represents the daily number of unique contacts a single individual in group $G_{k}$ has with individuals in group $G_{j}$, and $B$ is a scaling factor that can be used to set the reproduction number of the pathogen being considered (see Section 2.2). Since the initial phase of potential local outbreaks are the focus of this study, we did not account for depletion of susceptible hosts explicitly. The relative transmission rates from presymptomatic and asymptomatic individuals compared to symptomatic individuals are given by the scaled quantities $\eta \beta_{k j}$ and $\theta \beta_{k j}$, respectively, where $\eta$ and $\theta$ were chosen so that the proportions of transmissions generated by presymptomatic and asymptomatic hosts were in line with literature estimates (Ferretti et al., 2020). The parameter $\xi_{k}$ represents 
the proportion of asymptomatic infections in group $G_{k}$, so that a new infection in group $G_{k}$ either increases $A_{k}$ by one (with probability $\xi_{k}$ ) or increases $P_{k}$ by one (with probability $1-\xi_{k}$ ).

A presymptomatic individual in group $G_{k}$ may go on to develop symptoms (transition from $P_{k}$ to $S_{k}$ ) or be detected and isolated (so that $P_{k}$ decreases by one). A symptomatic individual in group $G_{k}$ may be detected and isolated as a result of successful surveillance, or may be removed due to self-isolation, recovery or death (so that $S_{k}$ decreases by one in either case). Similarly, an asymptomatic individual in group $G_{k}$ may be detected and isolated or recover (so that $A_{k}$ decreases by one). A schematic of the different possible events in the model is shown in Fig. 1.

The parameter $\lambda$ represents the rate at which presymptomatic individuals develop symptoms, so that the expected duration of the presymptomatic infectious period is $1 / \lambda$ days in the absence of surveillance of nonsymptomatic infected individuals. Similarly, the expected duration of the asymptomatic infectious period in the absence of surveillance is $1 / v$ days. The parameter $\mu$ represents the rate at which symptomatic individuals are removed as a result of self-isolation, recovery or death, so that the duration of time for which they are able to infect others is $1 / \mu$ days.

For each group $G_{k}$, the rate at which symptomatic individuals are detected and isolated as a result of enhanced surveillance is determined by the parameter $\rho_{k}$. Analogously, the parameter $\sigma_{k}$ governs the rate at which presymptomatic and asymptomatic individuals in $G_{k}$ are detected and isolated. We assumed that surveillance measures targeting nonsymptomatic hosts are equally effective for those who are presymptomatic and those who are asymptomatic, and therefore used the same rate of isolation due to surveillance for both of these groups.

\subsection{Reproduction number}

The effective reproduction number, $R$, represents the expected number of secondary infections generated by a single infected individual during their entire course of infection, accounting for interventions that are in place. Here, we take a heuristic approach to derive the following expression for $R$ :

$R=\sum_{k=1}^{16} \frac{N_{k}}{N} \sum_{l=1}^{16}\left[\frac{\xi_{k} \theta \beta_{k l}}{v+\sigma_{k}}+\left(1-\xi_{k}\right)\left(\frac{\eta \beta_{k l}}{\lambda+\sigma_{k}}+\frac{\lambda}{\lambda+\sigma_{k}} \frac{\beta_{k l}}{\mu+\rho_{k}}\right)\right]$ where $N=N_{1}+\cdots+N_{16}$ is the total population size. To obtain this expression, we first consider the expected number of secondary infections an infected individual in age group $G_{k}$ will generate in age group $G_{l}$. If an individual in age group $G_{k}$ experiences a fully asymptomatic course of infection, which occurs with probability $\xi_{k}$, they will generate new infections in age group $G_{l}$ at rate $\theta \beta_{k l}$ and recover or be isolated at rate $v+\sigma_{k}$. Therefore, the total number of infections they are expected to cause in age group $G_{l}$ is $\theta \beta_{k l} /\left(v+\sigma_{k}\right)$. If instead the individual in age group $G_{k}$ experiences a symptomatic course of infection, which occurs with probability $1-\xi_{k}$, whilst presymptomatic they will generate new infections in age group $G_{l}$ at rate $\eta \beta_{k l}$ and be isolated or develop symptoms at rate $\lambda+\sigma_{k}$. Thus, the total number of infections they are expected to cause in age group $G_{l}$ whilst presymptomatic is $\eta \beta_{k l} /\left(\lambda+\sigma_{k}\right)$. If they go on to develop symptoms before being isolated, which occurs with probability $\lambda /\left(\lambda+\sigma_{k}\right)$, applying similar reasoning they are expected to cause $\beta_{k l} /\left(\mu+\rho_{k}\right)$ new infections in age group $G_{l}$ whilst symptomatic. Combining these possibilities leads to the term in square brackets in expression (1), which is then summed over all possible age groups $G_{l}$ of the infectee. Finally, to obtain the full expression (1) we take a weighted average across all possible age groups $G_{k}$ of the infector, where the weights $N_{k} / N$ represent the proportions of the population belonging to each age group. This corresponds to the assumption that the initial infected host is more likely to belong to an age group containing more individuals than an age group with fewer individuals.

In the absence of interventions, i.e. when $\sigma_{k}=\rho_{k}=0$ (representing no enhanced isolation as a result of surveillance) and $\beta_{k l}$ is calculated using contact patterns that are characteristic of normal behaviour, the effective reproduction number, $R$, is equal to the basic reproduction number, $R_{0}$.

\subsection{Model parameterisation}

The numbers of individuals in each age group (values of $N_{k}$ ) were chosen according to United Nations age demographic data for the UK (United Nations, Department of Economic and Social Affairs, Population Division, 2019) (Fig. 2A). The daily numbers of contacts between individuals in each age group (values of $C_{k j}$ ) were set according to the $16 \times 16$ 'contact matrix' for the UK, in which the $(k, j)$ th entry represents the expected daily number of unique

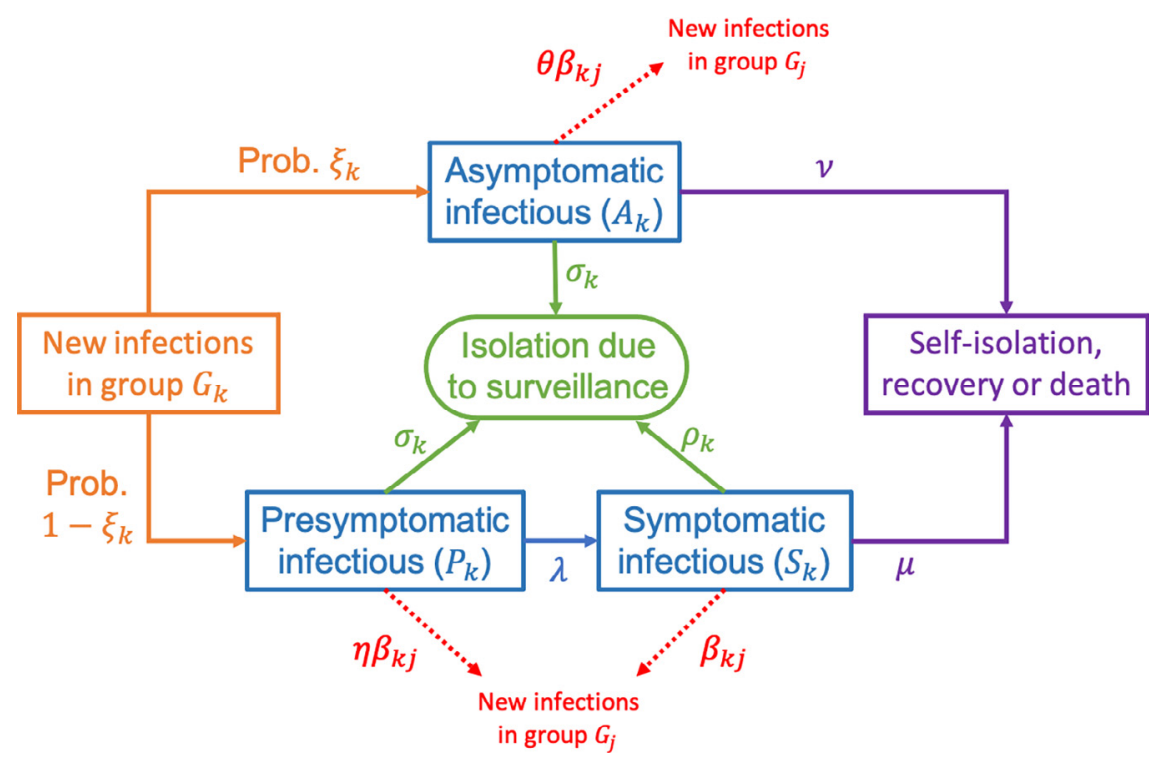

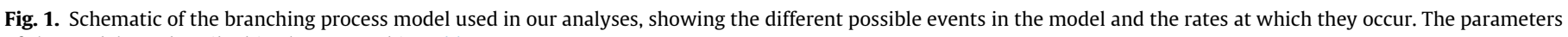
of the model are described in the text and in Tables 1-3. 
A.

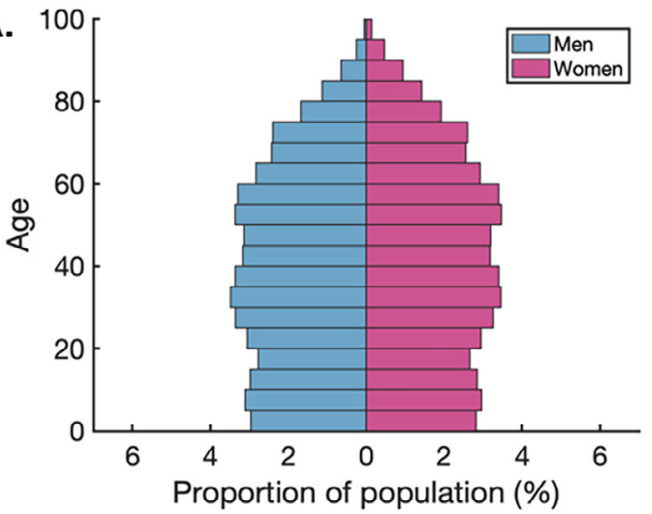

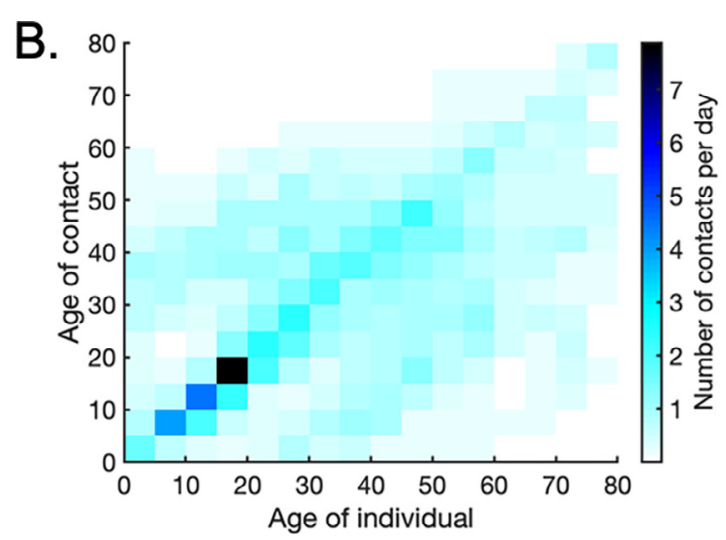
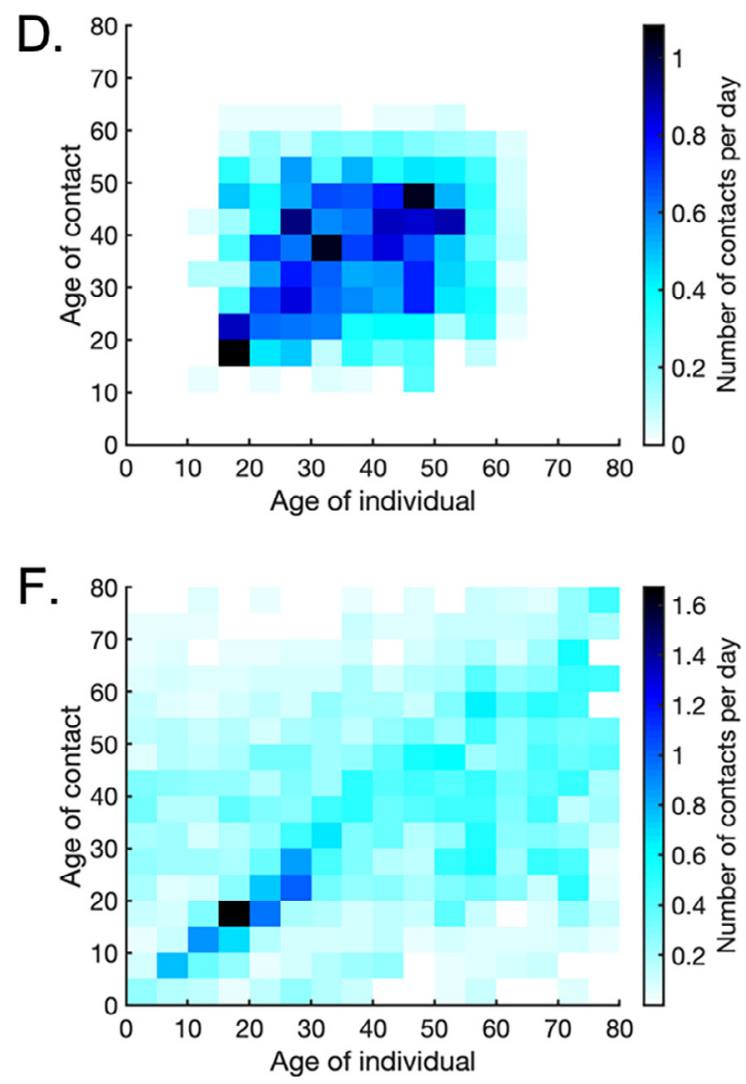

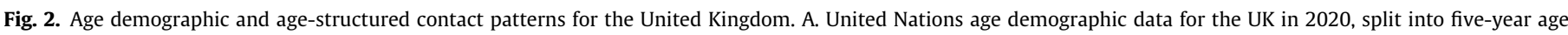

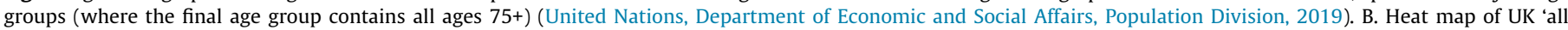

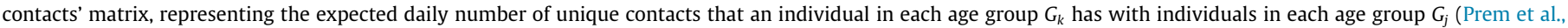

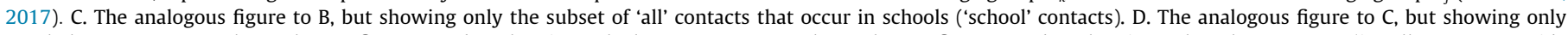

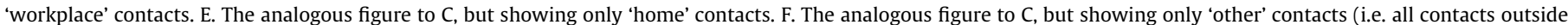
schools, workplaces or homes).

contacts an individual in age group $G_{k}$ has with individuals in age group $G_{j}$ (Prem et al., 2017). In addition to matrices representing 'all' contacts (Fig. 2B), we also considered matrices detailing a breakdown into 'school', 'work', 'home' and 'other' contacts (Fig. 2C-F), allowing us to investigate the effects of control interventions that reduce contacts in each of these settings.

Since we considered SARS-CoV-2 as a case study, we used studies conducted during the COVID-19 pandemic to inform the epidemiological parameters of our model. Despite previous research assessing the relationships between age and factors such as susceptibility to SARS-CoV-2 infection or the propensity to develop symptoms, there is some variation in estimated parameters between different studies. To test the robustness of our results to this uncertainty, we conducted our analyses under three different scenarios (A, B and C). In scenario A, we assumed that susceptibility to infection (values of $\omega_{k}$ ) and the proportion of hosts who experience a fully asymptomatic course of infection (values of $\xi_{k}$ ) are independent of age. In scenario B, susceptibility was assumed to vary with age but the proportion of asymptomatic infections is independent of age. In scenario $\mathrm{C}$, we allowed both susceptibility and the asymptomatic proportion to vary with age. The values used for the parameters $\omega_{k}$ and $\xi_{k}$ in each of these three scenarios are shown in Table 1 (see also Davies et al., 2020b).

In all scenarios considered, the inherent infectivity was not assumed to be age-dependent (i.e. $\tau_{k}=1$ for all values of $k$ ). In other words, the expected infectiousness of infected hosts in differ- 
Table 1

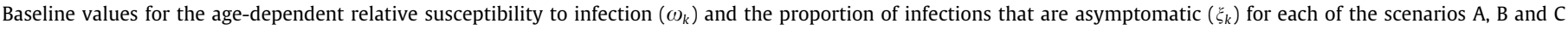
(Davies et al., 2020b).

\begin{tabular}{|c|c|c|c|c|}
\hline \multirow[t]{2}{*}{ Age group $\left(G_{k}\right)$} & \multicolumn{2}{|c|}{ Relative susceptibility $\left(\omega_{k}\right)$} & \multicolumn{2}{|l|}{ Asymptomatic proportion $\left(\xi_{k}\right)$} \\
\hline & Scenario A & Scenarios B \& C & Scenarios A \& B & Scenario C \\
\hline$G_{1}, G_{2}(0-9)$ & \multirow{8}{*}{1.0} & 0.4 & \multirow{8}{*}{$\begin{array}{l}0.584 \\
\text { (weighted average of age-dependent } \\
\text { values of } \xi_{k} \text { in scenario C) }\end{array}$} & 0.71 \\
\hline$G_{3}, G_{4}(10-19)$ & & 0.38 & & 0.79 \\
\hline$G_{5}, G_{6}(20-29)$ & & 0.79 & & 0.73 \\
\hline$G_{7}, G_{8}(30-39)$ & & 0.86 & & 0.67 \\
\hline$G_{9}, G_{10}(40-49)$ & & 0.8 & & 0.6 \\
\hline$G_{11}, G_{12}(50-59)$ & & 0.82 & & 0.51 \\
\hline$G_{13}, G_{14}(60-69)$ & & 0.88 & & 0.37 \\
\hline$G_{15}, G_{16}(70+)$ & & 0.74 & & 0.31 \\
\hline
\end{tabular}

Table 2

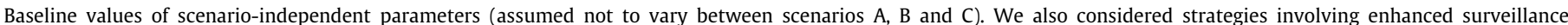
$\left(\rho_{k}, \sigma_{k}>0\right)-$ see Fig. 6.

\begin{tabular}{|c|c|c|c|}
\hline Parameter & Meaning & Baseline value & Justification \\
\hline$R_{0}$ & $\begin{array}{l}\text { Expected number of secondary infections generated by a } \\
\text { single infectious host in the absence of interventions }\end{array}$ & $R_{0}=3$ & $\begin{array}{l}\text { Within estimated range for original SARS-CoV-2 virus (Lai et al., 2020; } \\
\text { Liu et al., 2020; Zhai et al., 2020; Zhao et al., 2020) }\end{array}$ \\
\hline$\lambda$ & Rate at which presymptomatic hosts develop symptoms & $\lambda=1 / 2$ days $^{-1}$ & (Wei et al., 2020) \\
\hline$\mu$ & $\begin{array}{l}\text { Rate at which symptomatic hosts are removed due to self- } \\
\text { isolation, recovery or death }\end{array}$ & $\mu=1 / 8$ days $^{-1}$ & (Arons et al., 2020; Bullard et al., 2020; Wölfel et al., 2020) \\
\hline$v$ & $\begin{array}{l}\text { Rate at which asymptomatic hosts are removed due to } \\
\text { recovery or death }\end{array}$ & $v=1 / 10$ days $^{-1}$ & $\begin{array}{l}\text { Chosen so that, in the absence of interventions, the expected duration } \\
\text { of infection is identical for all infected hosts }\left(\frac{1}{v}=\frac{1}{\lambda}+\frac{1}{\mu}\right)\end{array}$ \\
\hline$\tau_{k}$ & (Relative) infectivity parameter & $\begin{array}{l}\tau_{k}=1 \text { for } \\
k=1, \cdots, 16\end{array}$ & Assumed \\
\hline$\rho_{k}$ & Isolation rate due to surveillance of symptomatic individuals & $\begin{array}{l}\rho_{k}=0 \text { for } \\
k=1, \cdots, 16\end{array}$ & $\mathrm{~N} / \mathrm{A}$ \\
\hline$\sigma_{k}$ & $\begin{array}{l}\text { Isolation rate due to surveillance of nonsymptomatic } \\
\text { individuals }\end{array}$ & $\begin{array}{l}\sigma_{k}=0 \text { for } \\
k=1, \cdots, 16\end{array}$ & $\mathrm{~N} / \mathrm{A}$ \\
\hline
\end{tabular}

ent age groups was governed solely by the proportion of asymptomatic infections in that age group. We chose the scaling factors $\eta$ and $\theta$ for the relative transmission rates from presymptomatic and asymptomatic individuals compared to symptomatic individuals so that the proportions of infections arising from each of these groups were in line with literature estimates (see Table 3 and Ferretti et al., 2020).

In the absence of enhanced isolation, we set the expected duration of the presymptomatic infectious period and the time for which symptomatic individuals are able to infect others to be $1 / \lambda=2$ days and $1 / \mu=8$ days, respectively (Wei et al., 2020; Arons et al., 2020; Bullard et al., 2020; Wölfel et al., 2020). The asymptomatic infectious period was then chosen so that all infected individuals are expected to be infectious for the same period (i.e. $1 / v=10$ days). In our initial analysis, we set the isolation rates $\rho_{k}$ and $\sigma_{k}$ equal to 0 for all $k$; later, we considered the effects of increasing these rates.

Initially, we fixed $R_{0}=3$ (in line with initial estimates of SARSCoV-2 transmissibility (Lai et al., 2020; Liu et al., 2020; Zhai et al., 2020; Zhao et al., 2020), before the emergence of more transmissible variants) and used expression (1) to determine the appropriate corresponding value of the scaling factor $B$. Later, when considering the impact of NPIs on the probability of a local outbreak, we retained this value of $B$ and used expression (1) to determine how the reproduction number changes as a result of the control implemented.

\subsection{Probability of a local outbreak}

The probability that an infected individual in a particular age group initiates a local outbreak when they are introduced into the population was calculated using the branching process model. One possible approach for approximating the age-dependent local outbreak probability using a branching process model is to run a large number of stochastic simulations of the model starting from a single infected individual in a particular age group, and record the proportion of simulations in which the pathogen does not fade out after only a small number of infections (Thompson et al., 2020). This would then need to be repeated for index cases of different ages. Here, we instead take an analytic approach, and derive a nonlinear system of simultaneous equations that determine the agedependent outbreak probabilities, as described below. The local

Table 3

Baseline values used for the scaling parameters $B, \eta$ and $\theta$ for each of the scenarios $\mathrm{A}, \mathrm{B}$ and $\mathrm{C}$.

\begin{tabular}{|c|c|c|c|c|c|}
\hline \multirow[t]{2}{*}{ Parameter } & \multirow[t]{2}{*}{ Meaning } & \multicolumn{3}{|l|}{ Value } & \multirow[t]{2}{*}{ Justification } \\
\hline & & $\begin{array}{l}\text { Scenario } \\
\text { A }\end{array}$ & $\begin{array}{l}\text { Scenario } \\
\text { B }\end{array}$ & $\begin{array}{l}\text { Scenario } \\
\text { C }\end{array}$ & \\
\hline$B$ & Transmission rate scaling factor & 0.0386 & 0.0559 & 0.0607 & $\begin{array}{l}\text { Chosen so that } R_{0}=3 \\
\text { (Lai et al., 2020; Liu et al., 2020; Zhai et al., 2020; Zhao et al., 2020) }\end{array}$ \\
\hline$\eta$ & $\begin{array}{l}\text { Relative transmission rate of presymptomatic } \\
\text { hosts compared to symptomatic hosts }\end{array}$ & 4.83 & 4.83 & 4.83 & $\begin{array}{l}\text { Chosen so that the proportion of all infections arising from } \\
\text { presymptomatic hosts is } 0.489 \text { (Ferretti et al., 2020) }\end{array}$ \\
\hline$\theta$ & $\begin{array}{l}\text { Relative transmission rate of asymptomatic } \\
\text { hosts compared to symptomatic hosts }\end{array}$ & 0.149 & 0.149 & 0.130 & $\begin{array}{l}\text { Chosen so that the proportion of all infections arising from entirely } \\
\text { asymptomatic hosts is } 0.106 \text { (Ferretti et al., 2020) }\end{array}$ \\
\hline
\end{tabular}


outbreak probabilities are then obtained by solving these equations numerically, and are analogous to the probabilities that would be derived from the simulation approach in the limit of infinitely many simulations. The benefit of our analytic approach is that it does not require a large number of stochastic simulations to be run.

The probability of a local outbreak not occurring (i.e. pathogen fadeout occurs), starting from a single symptomatic (or presymptomatic, asymptomatic respectively) infectious individual in age group $G_{k}$, was denoted by $x_{k}\left(y_{k}, z_{k}\right)$. Beginning with a single symptomatic individual in $G_{k}$, the possibilities for the next event are as follows:

1. The infected individual in $G_{k}$ infects a susceptible individual in $G_{j}$, so that either $A_{j}$ increases by one (with probability $\xi_{j}$ ) or $P_{j}$ increases by one (with probability $\left(1-\xi_{j}\right)$ ). This occurs with probability

$\alpha_{k j}=\frac{\beta_{k j}}{\mu+\rho_{k}+\sum_{l=1}^{16} \beta_{k l}}$.

2. The infected individual in $G_{k}$ recovers, dies or is isolated before infecting anyone else, so that $S_{k}$ decreases to zero (and there are no infected individuals left in the population). This occurs with probability

$\gamma_{k}=\frac{\mu+\rho_{k}}{\mu+\rho_{k}+\sum_{l=1}^{16} \beta_{k l}}$.

If there are no infectious hosts present in the population, then a local outbreak will not occur. Therefore, assuming that chains of transmission arising from infectious individuals are independent, the probability that no local outbreak occurs beginning from a single symptomatic individual in $G_{k}$ is

$x_{k}=x_{k}\left[\sum_{j=1}^{16} \alpha_{k j}\left(\xi_{j} z_{j}+\left(1-\xi_{j}\right) y_{j}\right)\right]+\gamma_{k}$.

Similarly, beginning instead with a single presymptomatic individual in $G_{k}$, the possibilities for the next event are:

1. The presymptomatic infected individual in $G_{k}$ infects a susceptible individual in $G_{j}$, so that (as before) either $A_{j}$ increases by one (with probability $\xi_{j}$ ) or $P_{j}$ increases by one (with probability $\left.\left(1-\xi_{j}\right)\right)$. This occurs with probability

$\tilde{\alpha}_{k j}=\frac{\eta \beta_{k j}}{\lambda+\sigma_{k}+\eta \sum_{l=1}^{16} \beta_{k l}}$.

2. The infected individual in $G_{k}$ develops symptoms (transitions from $P_{k}$ to $S_{k}$ ). This occurs with probability

$\delta_{k}=\frac{\lambda}{\lambda+\sigma_{k}+\eta \sum_{l=1}^{16} \beta_{k l}}$.

3. The infected individual in $G_{k}$ is isolated before infecting anyone else, so that $S_{k}$ decreases by one. This occurs with probability

$\phi_{k}=\frac{\sigma_{k}}{\lambda+\sigma_{k}+\eta \sum_{l=1}^{16} \beta_{k l}}$.

Therefore, the probability that no local outbreak occurs beginning from a single presymptomatic individual in $G_{k}$ is

$y_{k}=y_{k}\left[\sum_{j=1}^{16} \tilde{\alpha}_{k j}\left(\xi_{j} z_{j}+\left(1-\xi_{j}\right) y_{j}\right)\right]+\delta_{k} x_{k}+\phi_{k}$.
Similarly, the probability $z_{k}$ that a local outbreak does not occur starting from a single asymptomatic individual in $G_{k}$ satisfies the equation

$z_{k}=z_{k}\left[\sum_{j=1}^{16} \hat{a}_{k j}\left(\xi_{j} z_{j}+\left(1-\xi_{j}\right) y_{j}\right)\right]+\epsilon_{k}$,

where

$\hat{\alpha}_{k j}=\frac{\theta \beta_{k j}}{v+\sigma_{k}+\theta \sum_{l=1}^{16} \beta_{k l}}$ and $\epsilon_{k}=\frac{v+\sigma_{k}}{v+\sigma_{k}+\theta \sum_{l=1}^{16} \beta_{k l}}$.

The system of simultaneous Eqs. (2)-(4) can be solved numerically to obtain $x_{k}, y_{k}$ and $z_{k}$ (here, we did this using the MATLAB nonlinear system solver 'fsolve'). Specifically, we take the minimal non-negative solution, as is standard when calculating extinction probabilities using branching process models (Lovell-Read et al., 2021 ; Norris, 1997). Then, for each $k$, the probability of a local outbreak occurring beginning from a single symptomatic (or presymptomatic, asymptomatic respectively) individual in group $G_{k}$ is given by $1-x_{k}\left(1-y_{k}, 1-z_{k}\right)$.

Throughout, we consider the probability $p_{k}$ of a local outbreak occurring beginning from a single nonsymptomatic individual in group $G_{k}$ arriving in the population at the beginning of their infection:

$p_{k}=\xi_{k}\left(1-z_{k}\right)+\left(1-\xi_{k}\right)\left(1-y_{k}\right)$.

The average local outbreak probability, $P$, which is defined as the probability of a local outbreak when the index case is chosen randomly from the population, is also considered. The value of $P$ is therefore a weighted average of the $p_{k}$ values, where the weights correspond to the proportion of the population represented by each group:

$P=\frac{1}{N} \sum_{k=1}^{16} N_{k} p_{k}$

This reflects an assumption that the index case is more likely to come from an age group with more individuals than an age group with fewer individuals.

All computing code used to implement the above methods was written in MATLAB version R2019a, and is available at https:// github.com/francescalovellread/age-dependent-outbreak-risks.

\section{Results}

\subsection{Effect of the age of the index case on the risk of a local outbreak}

We first considered the probability that a single infected individual in a particular age group $G_{k}$ initiates a local outbreak when introduced into a new host population. This quantity was calculated for each of the three scenarios A, B and C (Fig. 3).

In scenario $A$, the variation in the local outbreak risk for introduced cases of different ages is driven solely by the numbers of contacts between individuals. As a result, due to their higher numbers of daily contacts, school- and working-age individuals are more likely to trigger a local outbreak than children under five or adults over 60 , with index cases aged $15-19$ posing the highest risk (Fig. 3A). These findings do not change significantly when susceptibility is allowed to vary with age in scenario B (Fig. 3B). However, in scenario $C$, assuming that the clinical fraction also varies between age groups alters the age-dependent risk profile substantially. This is because asymptomatic individuals are assumed to be less infectious than symptomatic individuals, and therefore an index case in an age group with a high proportion of asymptomatic infections is less likely to initiate a local outbreak. In this scenario, index cases aged 40 or over had a disproportionately high probabil- 

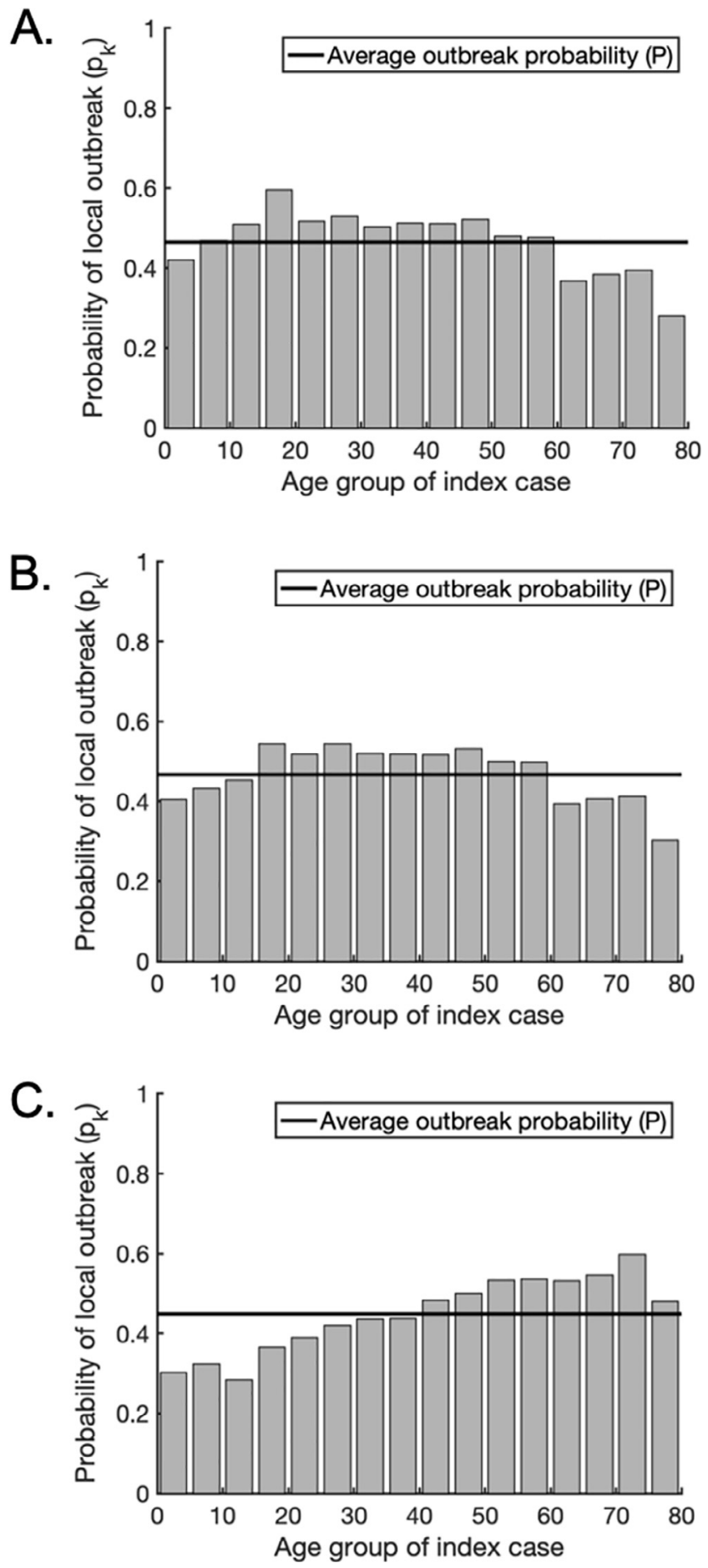

Fig. 3. The probability of a local outbreak depends on the age of the index case. A. The probability that a single infected individual in any given age group triggers a local outbreak (grey bars) for scenario A, in which susceptibility and clinical fraction are assumed constant across all age groups. The weighted average local outbreak probability $P$ is shown by the black horizontal line. B. The analogous figure to A but for scenario $B$, in which clinical fraction is assumed constant across all age groups but susceptibility varies with age (Table 1 ). C. The analogous figure to A but for scenario $C$, in which both susceptibility and clinical fraction vary with age (Table 1 ).

ity of generating a local outbreak, with individuals aged 70-74 presenting the highest risk (Fig. 3C). These individuals are more likely to develop symptoms than younger individuals (Table 1), leading to a higher expected infectiousness. In contrast, individuals under the age of 40 had a below average probability of generating a local outbreak, with individuals aged 10-14 presenting the lowest risk. Noticeably, individuals aged 5-19 presented relatively low risks, despite the high numbers of contacts occurring among these age groups (Fig. 2B). In this scenario, the large number of contacts was offset by the fact that individuals in these age groups are more likely to be asymptomatic and consequently less infectious than older individuals (Table 1). Therefore, an index case in one of these age groups is likely to lead to fewer secondary transmissions. Furthermore, the contact patterns between individuals in these age groups are highly assortative with respect to age (Fig. 2B). Therefore, in addition to the index case being less infectious, a high proportion of the contacts they make are with individuals who are also likely to be less infectious, as well as being less susceptible to infection in the first place.

We performed our subsequent analyses for each of the three scenarios A, B and C, with qualitatively similar results. The figures shown in the main text are for scenario $C$, since we deem this scenario to be the most realistic for SARS-CoV-2 transmission, but the analogous results for scenarios A and B are presented in Supplementary Fig. S1-6.

\subsection{Effect of the target age group on NPI effectiveness}

We next considered the effects of NPIs that reduce the number of contacts between individuals on the probability that an introduced case will lead to a local outbreak. To approximate the relative effects of school closures, workplace closures and population-wide social distancing policies, we calculated the agedependent risk profiles when each of these types of contact were excluded from the overall contact matrix.

First, we removed all 'school' contacts from the total contact matrix (Fig. 4A). For scenario C, removing 'school' contacts led to a $4.2 \%$ reduction in the average probability of a local outbreak (from 0.449 to 0.430 ). This small reduction is unsurprising for scenario $C$, since in that scenario school-aged infected individuals are assumed to be more likely to be asymptomatic than other infected individuals, and therefore their expected infectiousness is lower. However, even for scenarios A and B, in which schoolaged individuals present the greatest risk of triggering a local outbreak, the effectiveness of removing 'school' contacts alone at reducing the local outbreak probability was limited (reductions of $7.2 \%$ and $4.75 \%$ respectively; see Supplementary Figs S1A, $\mathrm{S} 4 \mathrm{~A}$ ). In each scenario, the reduction in risk was predominantly for school-aged index cases, with the risk from index cases of other ages only slightly reduced. Second, we considered the effects of removing 'work' contacts from the total contact matrix (Fig. 4B). This led to a more substantial $25.4 \%$ reduction in the average probability of a local outbreak for scenario $C$ (with corresponding reductions of $19.0 \%$ and $24.0 \%$ for scenarios A and B respectively; see Supplementary Figs S1B, S4B). As well as reducing the risk of a local outbreak from an index case of working age, removing 'work' contacts also reduced the probability of a local outbreak occurring starting from a school-aged individual. This is because closing workplaces helps to block chains of transmission that begin with an infected child. For example, a transmission chain involving a child transmitting to an adult at home, followed by subsequent spread around the adult's workplace, will be less likely to occur. Third, we investigated the effect of removing all 'other' contacts, reflecting perfect social distancing being observed outside of the home, school or workplace (Fig. 4C). This had the most significant effect of the three types of contactreducing intervention considered, reducing the probability of a local outbreak by $41.7 \%$ for scenario $C$ (and $30.7 \%$ or $33.2 \%$ for scenarios A and B, respectively).

In the three cases described above, we considered complete reductions in 'school', 'work' and 'other' contacts, respectively. 
A.

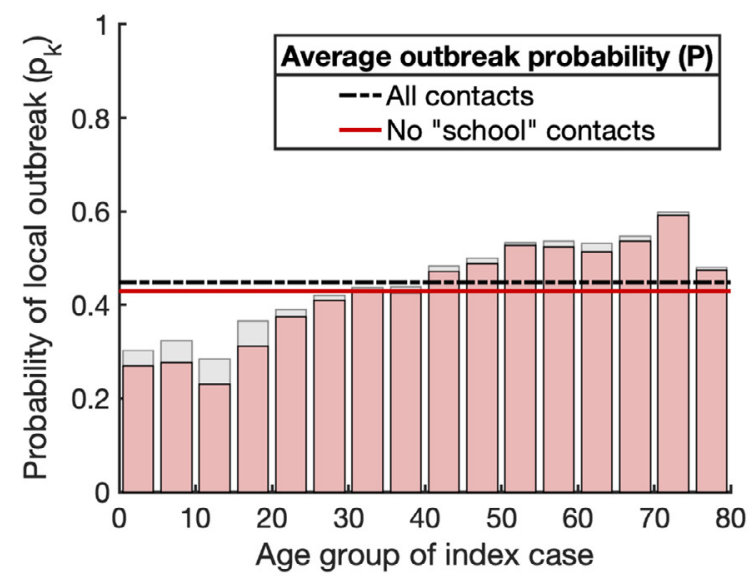

C.

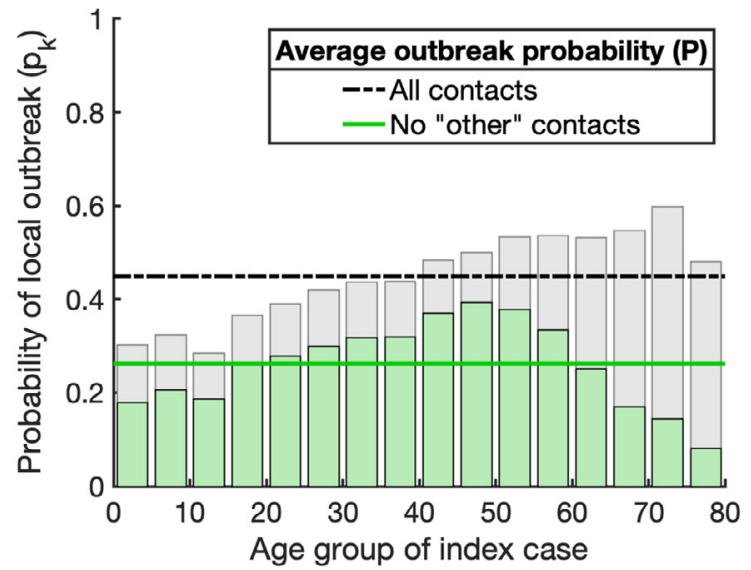

B.

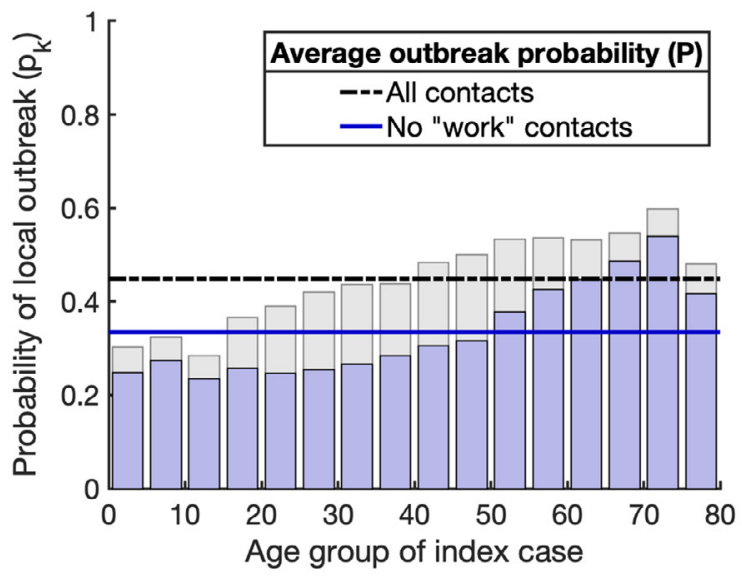

D.

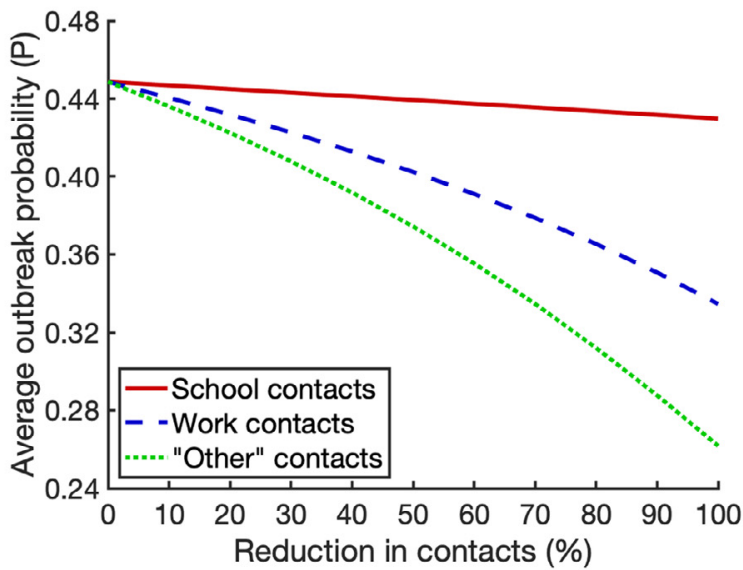

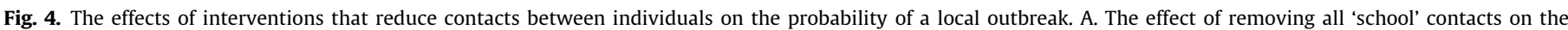

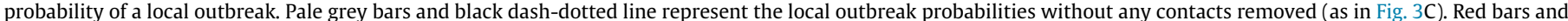

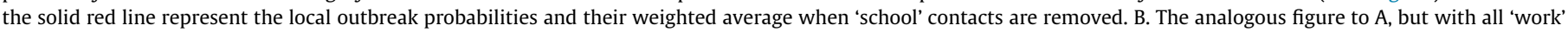

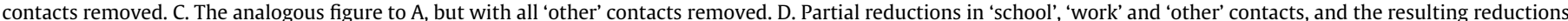

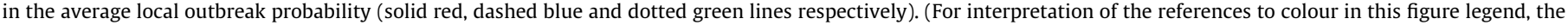
reader is referred to the web version of this article.)

In practice, such complete elimination of contacts is unlikely. We therefore also considered partial reductions in 'school', 'work' and 'other' contacts, and compared the resulting reductions in the local outbreak probability (Fig. 4D). For any given percentage reduction in contacts, reducing 'other' contacts always led to the largest reduction in the local outbreak probability (see also Supplementary Figs S1D, S4D). This suggests that reducing social contacts outside schools and workplaces can be an important component of strategies to reduce the risk of local outbreaks of SARS-CoV-2. However, this alone is not enough to eliminate the risk of local outbreaks entirely. For greater risk reductions using contact-reducing NPIs, a mixed approach involving combinations of reductions in 'school', 'workplace' and 'other' contacts is needed.

\subsection{Mixed strategies for reducing the local outbreak risk}

Next, we considered the effects of combining reductions in 'school', 'work' and 'other' contacts on the local outbreak probability (Fig. 5; analogous results for scenarios A and B are shown in Supplementary Figs S2 and S5). We allowed reductions in 'school' and 'work' contacts to vary between $0 \%$ and $100 \%$ whilst 'other' contacts were reduced by $25 \%, 50 \%$ or $75 \%$ (Fig. 5A,B,C, respectively).
Since NPIs have negative economic, social and non-disease health consequences, policy makers may choose to implement public health measures in which the risk of local outbreaks is not eliminated completely. These results provide contact reduction targets for mixed strategies in which the local outbreak probability is reduced to a pre-specified 'acceptable' level. For example, to reduce the local outbreak probability to 0.25 , 'other' contacts could be reduced by $25 \%$ from the baseline level, and 'school' and 'home' contacts reduced as indicated by the red dotted contour marked '0.25' in Fig. 5A. Alternatively, to achieve the same local outbreak risk, 'other' contacts can instead be reduced by $50 \%$ or by $75 \%$ with the degree of 'school' and 'work' reductions chosen according to the contours marked ' $0.25^{\prime}$ in Fig. $5 B, C$ respectively.

If a policy maker wishes to eliminate the local outbreak risk entirely using contact-reducing NPIs, for the model parameterisation considered very significant reductions in multiple types of contacts are needed in combination. For example, even if all 'school' and 'work' contacts are removed, 'other' contacts must be reduced by $66 \%$ for the overall average local outbreak probability to fall below 0.01 (Fig. 5D). Since such substantial reductions in multiple types of contacts are unlikely to be possible, this suggests that contact-reducing NPIs must be combined with other interventions, such as effective surveillance and isolation strategies, to eliminate local outbreaks. 
A.

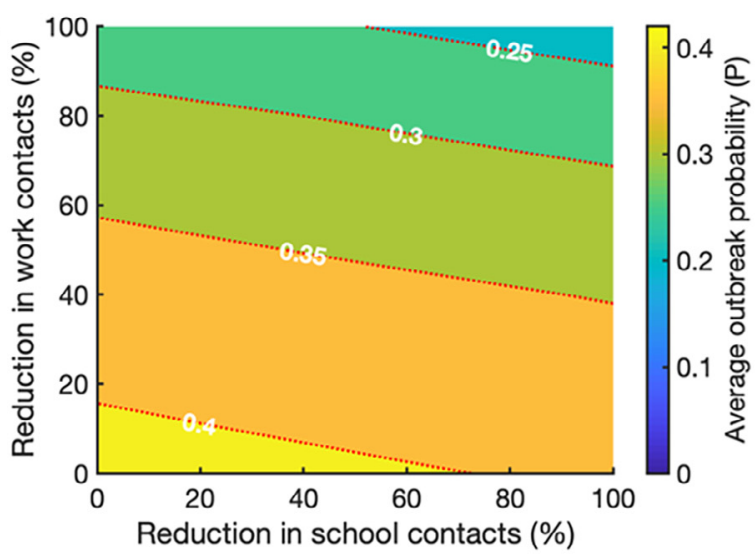

B.

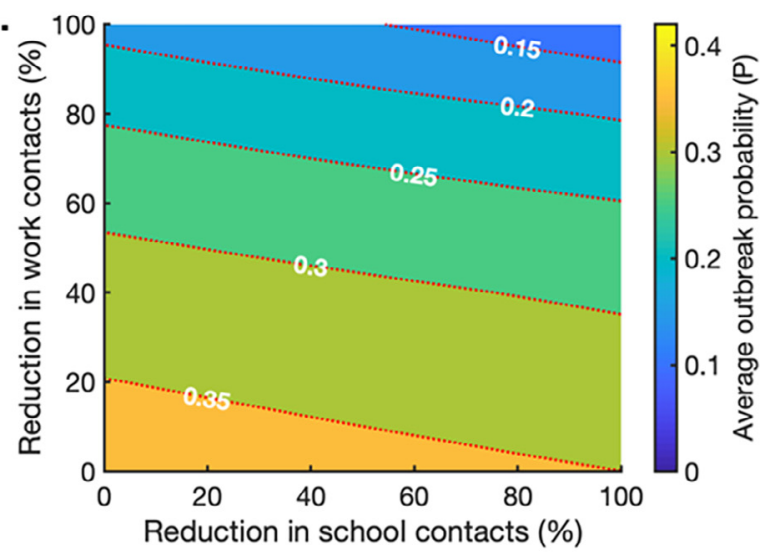

D.

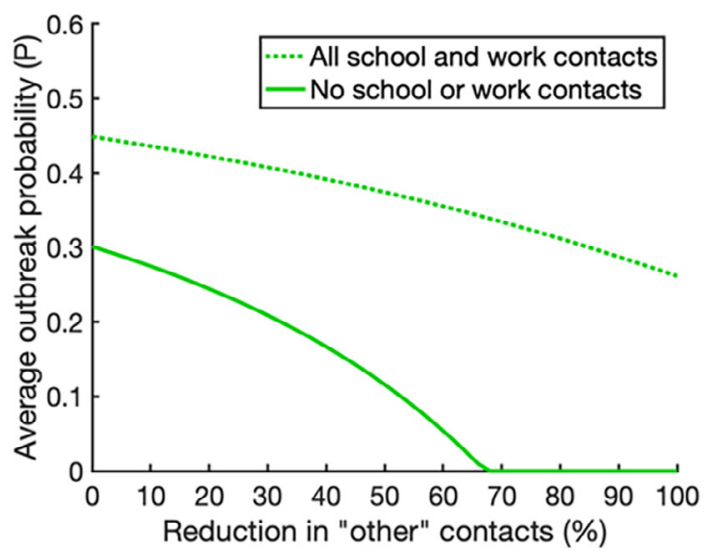

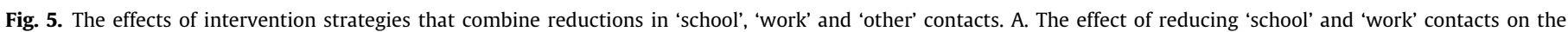

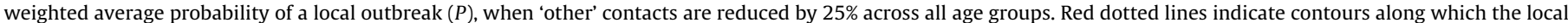

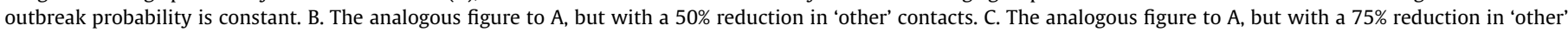

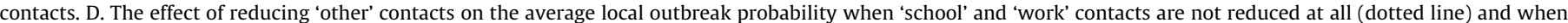

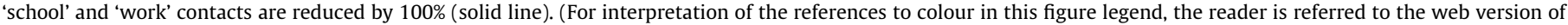
this article.)

\subsection{Effect of surveillance on the contact-reducing NPIs required for local outbreak control}

We considered whether or not low local outbreak probabilities can be achieved using limited contact-reducing NPIs in combination with other interventions. Specifically, the effects of surveillance and isolation of infected individuals (through e.g. contact tracing) as well as reducing contacts in schools, workplaces and other locations, were assessed. While results are shown for scenario C in Fig. 6, analogous results for scenarios A and B are presented in Supplementary Figs S3 and S6.

Initially, we considered the effect of increasing the rate at which symptomatic and/or nonsymptomatic infected individuals are detected and isolated as a result of surveillance, in the absence of contact-reducing NPIs (i.e. with no reduction in the number of contacts between individuals compared to the baseline case in Fig. 2B) (Fig. 6A,B). For symptomatic hosts, this represents an enhanced rate of isolation compared to the baseline rate of self-isolation already present in the model. Isolation of nonsymptomatic hosts was more effective at reducing the local outbreak probability than isolation of symptomatic hosts (Fig. 6A,B), although of course this is more challenging to achieve (Lovell-Read et al., 2021). However, if fast isolation of nonsymptomatic hosts could be achieved through efficient large-scale testing (potentially in combination with contact tracing (Hart et al., 2021)), the probability of local outbreaks could be reduced substantially through this measure alone.

We then demonstrated the effects of combining contactreducing NPIs with enhanced isolation of infected hosts due to infection surveillance. First, we increased the enhanced isolation rate of symptomatic individuals to $\rho_{k}=1 / 2$ days $^{-1}$. In the absence of other interventions, this reduced the local outbreak probability by $22.0 \%$ (Fig. 6C). With this level of surveillance, the local outbreak risk could be reduced below 0.01 with a reduction in 'work' and 'other' contacts of around $73 \%$ each, for example.

Finally, keeping the enhanced isolation rate of symptomatic individuals equal to $\rho_{k}=1 / 2$ days $^{-1}$, we increased the isolation rate of nonsymptomatic individuals to $\sigma_{k}=1 / 7$ days $^{-1}$. In this case, the local outbreak probability without contact-reducing NPIs fell by $59.4 \%$ compared to a situation without enhanced surveillance (Fig. 6E), and the reductions in 'work' and 'other' contacts needed to bring the local outbreak probability below 0.01 were significantly smaller (Fig. 6F). For example, if 'work' contacts can be reduced by $50 \%$, then 'other' contacts only need to be reduced by $43 \%$. This indicates that effective surveillance of both symptomatic and nonsymptomatic individuals can substantially lower the extent of contact-reducing NPIs that are required to achieve substantial reductions in local outbreak risks. 
A.

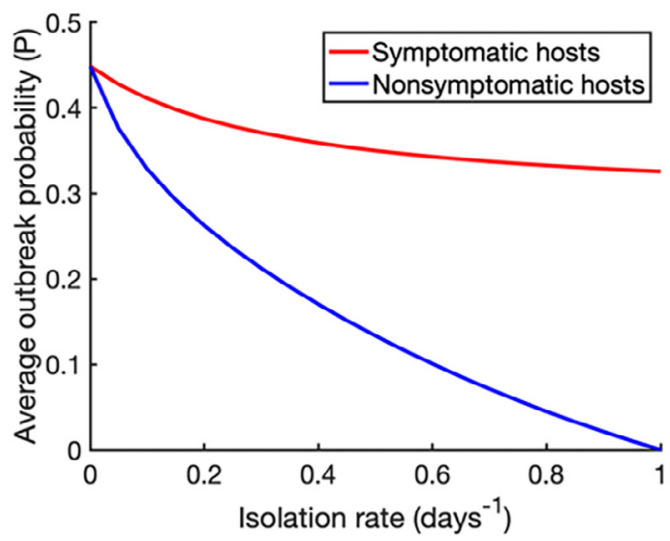

C.

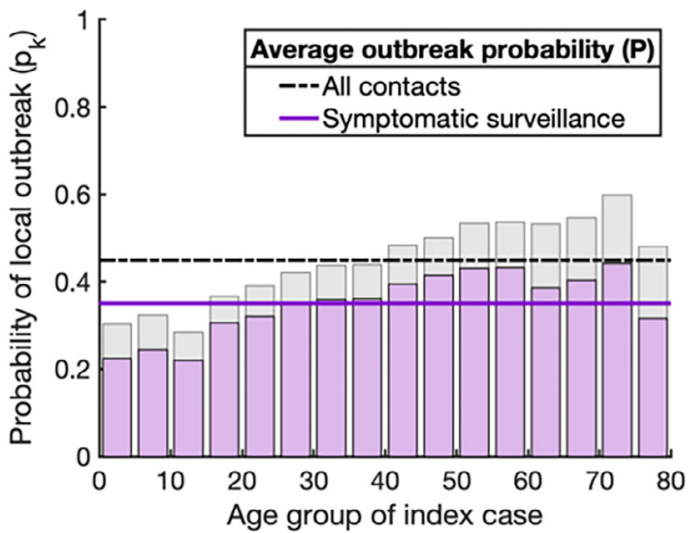

E

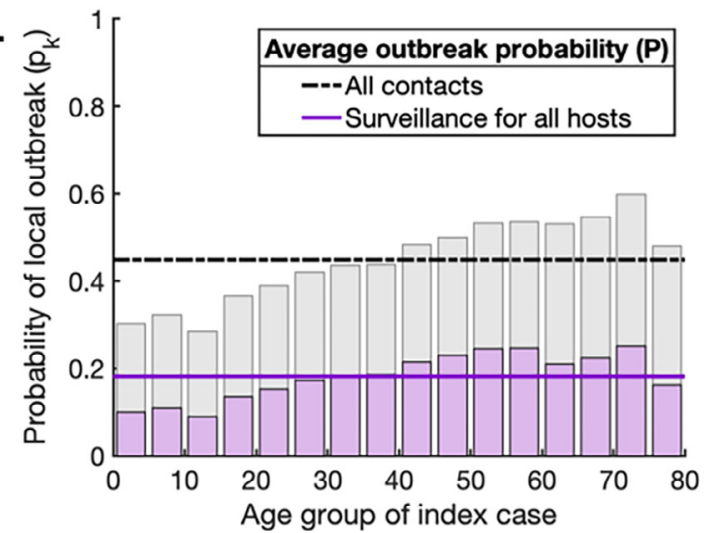

B.

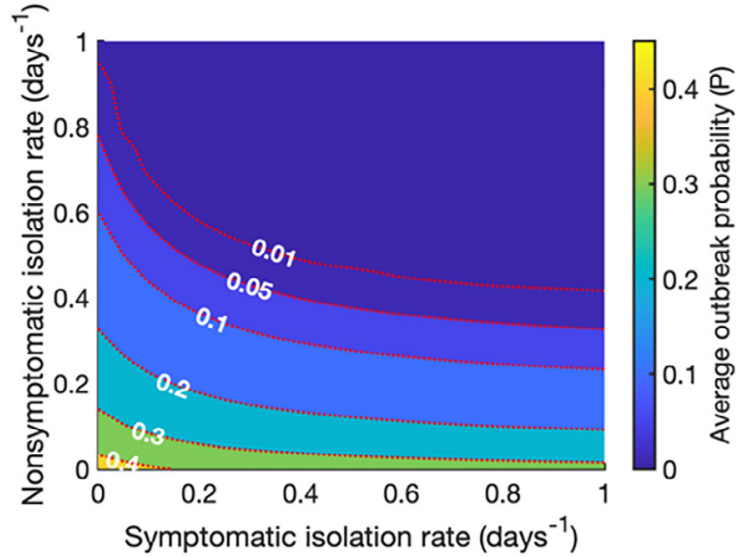

D.

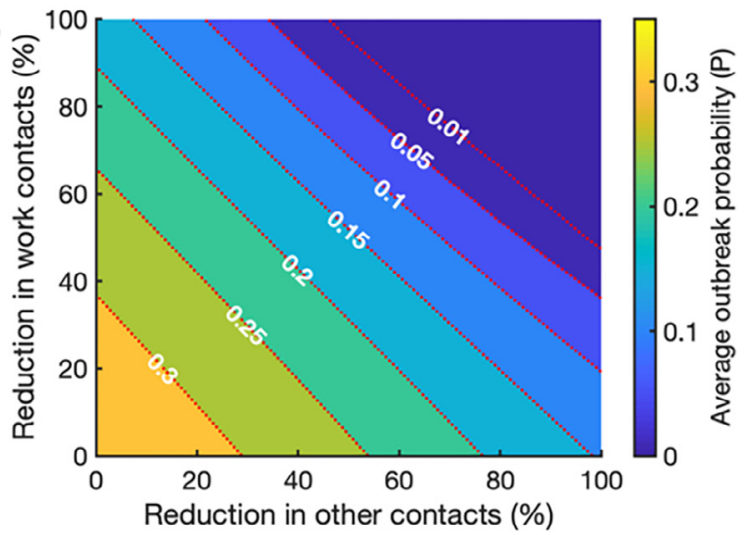

$\mathrm{F}$.

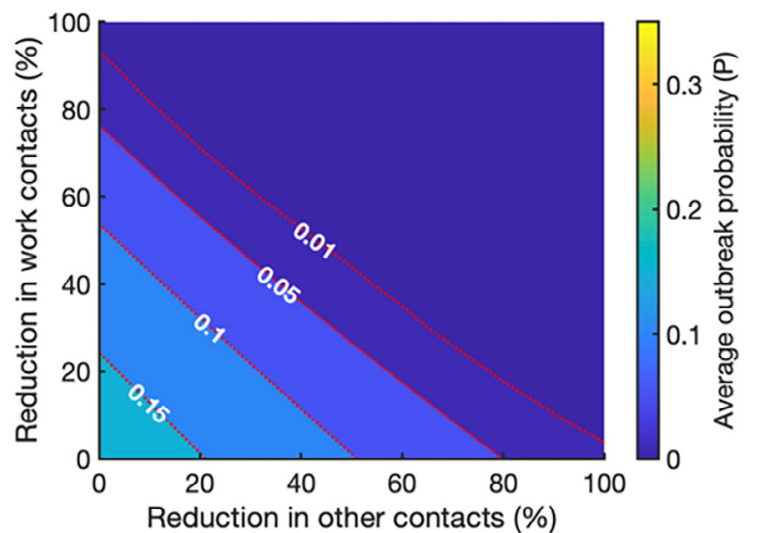

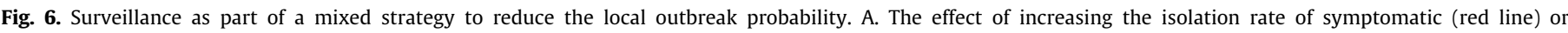

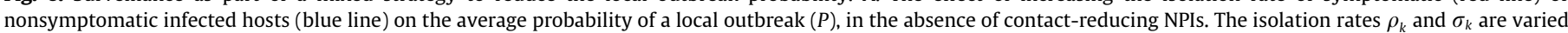

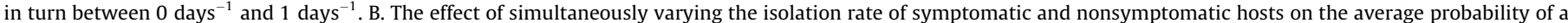

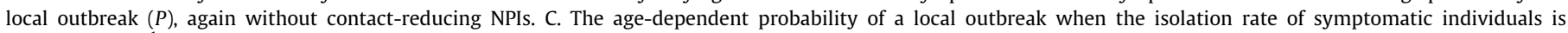

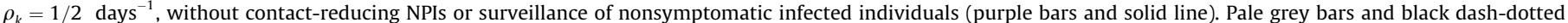

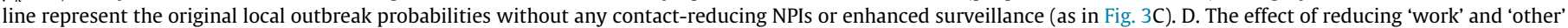

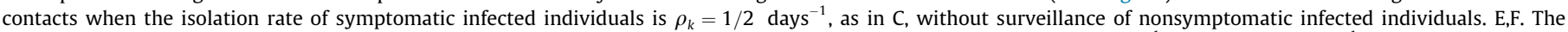

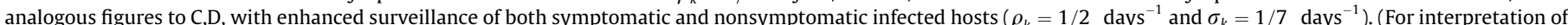
the references to colour in this figure legend, the reader is referred to the web version of this article.)

\section{Discussion}

During the COVID-19 pandemic, public health measures that reduce the numbers of contacts between individuals have been implemented in countries globally. These measures include school closures, workplace closures and population-wide social distancing policies. Contact-reducing NPIs have been shown to be effective at reducing SARS-CoV-2 transmission, and have also been used previ- ously during influenza pandemics (Hatchett et al., 2007; Huang et al., 2021; Flaxman et al., 2020; Borse et al., 2011). However, long-term implementation of these measures has negative social, psychological and economic consequences (Berkman, 2008; Donohue and Miller, 2020; Silverman et al., 2020; Sadique et al., 2008; Brooks et al., 2020; Loades et al., 2020). It is therefore important to assess the effectiveness of different contact-reducing NPIs at lowering transmission and preventing local outbreaks, in order 
to design effective targeted control strategies that avoid unnecessarily strict measures.

Here, we constructed a branching process model to estimate the risk of local outbreaks under different contact-reducing NPIs and different levels of surveillance for symptomatic and nonsymptomatic infected individuals. Unlike previous approaches for estimating outbreak risks using branching processes (Althaus et al., 2015; Thompson et al., 2019; Thompson, 2020; Hellewell et al., 2020; Lovell-Read et al., 2021; Nishiura et al., 2011), we considered the effects of age-related heterogeneities affecting transmission for infected individuals of a wide range of ages, including agedependent variations in social mixing patterns, susceptibility to infection and clinical fraction. Using SARS-CoV-2 as a case study, we demonstrated that the risk that an introduced case initiates a local outbreak depends on these age-related factors and on the age of the introduced case (Fig. 3), as well as the age-structure of the local population.

We used our model to assess the effects of reducing the numbers of contacts that occur in school, in the workplace and elsewhere. Of the three contact-reducing NPIs considered, removing 'school' contacts had the smallest effect on the probability of observing a local outbreak, even when age-dependent variations in susceptibility and clinical fraction were ignored (Fig. 4A,D, Supplementary Figs S1A,D and S4A,D). This can be attributed to the fact that school closures predominantly reduce contacts between individuals aged 5-19, who only account for approximately $23 \%$ of the total population (United Nations, Department of Economic and Social Affairs, Population Division, 2019). Additionally, these individuals tend to have large numbers of contacts outside of the school environment (Fig. 2E,F). Therefore, compared to other measures, interrupting within-school transmission may have only a limited effect on transmission in the wider population, particularly when school-aged individuals are less susceptible to infection and more likely to experience subclinical courses of infection. In contrast, reducing contacts that occur outside schools or workplaces was the most effective intervention, significantly lowering the local outbreak risk across all age groups, and for those aged over 60 in particular (Fig. 4C,D, and Supplementary Figs S4C,D). This could explain the success of social distancing strategies worldwide for reducing observed COVID-19 cases and deaths.

Mixed strategies combining reductions in 'school', 'work' and 'other' contacts led to greater reductions in the local outbreak probability than individual interventions (Fig. 5A-C), but very large reductions in all three types of contact were required to eliminate the risk of local outbreaks entirely (Fig. 5D). However, implementing effective surveillance to identify infected hosts (followed by isolation) led to substantial reductions in the risk of local outbreaks even in the absence of other control measures (Fig. 6A,B). In the scenarios considered here, with an efficient surveillance strategy in place, significantly smaller reductions in 'work' and 'other' contacts were needed to render the local outbreak probability negligible, even when 'school' contacts were not reduced at all (Fig. 6C-F). This supports the use of surveillance that targets both symptomatic and nonsymptomatic individuals, such as contact tracing and isolation strategies or population-wide diagnostic testing, to help prevent local outbreaks (Lovell-Read et al., 2021).

Although here we used SARS-CoV-2 as a case study, our model provides a framework for estimating the risk of local outbreaks in age-structured populations that can be adapted for other pathogens, provided sufficient data are available to parametrise the model appropriately. The effects of age-structure on local outbreak risks may vary for pathogens with different epidemiological characteristics. For influenza-A viruses, for example, susceptibility to infection tends to decrease with age, whilst the risk of an infection leading to severe symptoms is greater both for the elderly and for the very young (Davies et al., 2020b; Greer et al., 2010; Clohisey and Baillie, 2019). This is in contrast to SARS-CoV-2, for which children are more likely to experience subclinical courses of infection. In this study, we used age demographic and contact data for the UK, but equivalent data for other countries are available and can easily be substituted into our model to estimate outbreak risks elsewhere (Prem et al., 2017; United Nations, Department of Economic and Social Affairs, Population Division., 2019).

One caveat of the results for SARS-CoV-2 presented here is that, although the epidemiological parameters of our model were chosen to be consistent with reported literature estimates, there is considerable variation between studies. In particular, the precise age-dependent variation in susceptibility and clinical fraction remains unclear, and the relative infectiousness of asymptomatic, presymptomatic and symptomatic hosts has not been determined exactly. Furthermore, the inherent transmissibility of SARS-CoV-2 is now higher than in the initial stage of the pandemic, due to the appearance of novel variants. To explore ongoing local outbreak risks due to SARS-CoV-2, it would be necessary to update the model to reflect the increased transmissibility of the Delta variant (Campbell et al., 2021). Due to the uncertainty in model parameter values, we conducted sensitivity analyses to explore the effects of varying the parameters of the model on our results (Supplementary Figs S1-12). In each case that we considered, our main conclusions were unchanged: the probability that an introduced case initiates a local outbreak depends on age-dependent factors affecting pathogen transmission and control, with widespread interventions and combinations of NPIs reducing the risk of local outbreaks most significantly.

An important limitation of our approach to modelling contactreducing NPIs is that we made a standard assumption in our main analyses that 'school', 'work' and 'other' contacts are independent (Zhang et al., 2020; Davies et al., 2020b; Davies et al., 2020a). In other words, reducing the numbers of contacts in one location did not affect the numbers of contacts occurring in another. In reality, this is unlikely to be the case. For example, closing schools also affects workplace contacts, as adults may then work from home in order to fulfil childcare requirements. Additionally, the contact data that we used represent the number of unique contacts per day and do not reflect the numbers of repeated contacts with the same person, which affect the risk of transmission between individuals. These assumptions could in principle be removed, if relevant data become available - for example, data describing the effects of school closures on numbers of contacts in other locations. To demonstrate how changes in multiple types of contact due to NPIs could be implemented in the model, we conducted a supplementary analysis in which we investigated the effects of removing all 'school' contacts and allowing for concurrent changes in 'work', 'home' and 'other' contacts (Supplementary Figs S13-14). These results support our conclusion that school closures are unlikely to have a substantial impact on SARS-CoV-2 transmission when applied as the sole NPI. The benefit of school closures could even potentially be outweighed by the possible secondary effects on other types of contacts. An improved understanding of how NPIs affect different types of contact is important for more accurate assessments of interventions in future.

Despite the simplifications made, our model provides a useful framework for estimating the risk of local outbreaks and the effects of NPIs. Different measures can be considered in combination in the model to develop strategies for lowering local outbreak risks. Our results emphasise the importance of quantifying agedependent factors that affect transmission dynamics, such as susceptibility to infection and the proportion of hosts who develop clinical symptoms, for individuals of different ages. As we have shown, it is crucial to take age-dependent factors into account when assessing local outbreak risks and designing public health measures. 


\section{Funding}

FALR acknowledges funding from the Biotechnology and Biological Sciences Research Council (BBSRC), grant number BB/ M011224/1. SS was funded by a BBSRC Research Experience Placement and a Rokos award for undergraduate research from Pembroke College Oxford. RNT was supported by UK Research and Innovation (UKRI) via grant EP/V053507/1. The funders had no role in the study design; the collection, analysis and interpretation of data; the writing of the report; or the decision to submit the article for publication.

\section{CRediT authorship contribution statement}

Francesca A. Lovell-Read: Conceptualization, Methodology, Investigation, Writing - original draft, Writing - review \& editing. Silvia Shen: Conceptualization, Investigation, Writing - review \& editing. Robin N. Thompson: Conceptualization, Methodology, Writing - original draft, Writing - review \& editing, Supervision.

\section{Data availability}

All computer code used in this paper was written in MATLAB version R2019a, and is available at the following GitHub repository: https://github.com/francescalovellread/age-dependent-outbreak-risks.

\section{Declaration of Competing Interest}

The authors declare that they have no known competing financial interests or personal relationships that could have appeared to influence the work reported in this paper.

\section{Appendix A. Supplementary data}

Supplementary data to this article can be found online at https://doi.org/10.1016/j.jtbi.2021.110983.

\section{References}

Althaus, C.L., Low, N., Musa, E.O., Shuaib, F., Gsteiger, S., 2015. Ebola virus disease outbreak in Nigeria: transmission dynamics and rapid control. Epidemics 11, 80-84. https://doi.org/10.1016/j.epidem.2015.03.001.

Arons, M.M., Hatfield, K.M., Reddy, S.C., Kimball, A., James, A., Jacobs, J.R., Taylor, J., Spicer, K., Bardossy, A.C., Oakley, L.P., et al., 2020. Presymptomatic SARS-CoV-2 infections and transmission in a skilled nursing facility. N. Engl. J. Med. 382, 2081-2090. https://doi.org/10.1056/NEJMoa2008457.

Béraud, G., Kazmercziak, S., Beutels, P., Levy-Bruhl, D., Lenne, X., Mielcarek, N., Yazdanpanah, Y., Boëlle, P.-Y., Hens, N., Dervaux, B., Chuang, J.-H., 2015. The French connection: the first large population-based contact survey in France relevant for the spread of infectious diseases e0133203 PLoS ONE 10 (7). https://doi.org/10.1371/journal.pone.0133203.

Berkman, B.E., 2008. Mitigating Pandemic Influenza: The Ethics of Implementing a School Closure Policy. J. Public Health Manag. Pract. 14, 372-378. https://doi. org/10.1097/01.PHH.0000324566.72533.0b.

Borse, R.H., Behravesh, C.B., Dumanovsky, T., Zucker, J.R., Swerdlow, D., Edelson, P., Choe-Castillo, J., Meltzer, M.I., 2011. Closing Schools in Response to the 2009 Pandemic Influenza A H1N1 Virus in New York City: Economic Impact on Households. Clin. Infect. Dis. 52, S168-S172. https://doi.org/ 10.1093/cid/ciq033.

Brooks, S.K., Webster, R.K., Smith, L.E., Woodland, L., Wessely, S., Greenberg, N., Rubin, G.J., 2020. The psychological impact of quarantine and how to reduce it: rapid review of the evidence. The Lancet 395, 912-920. https://doi.org/10.1016/ S0140-6736(20)30460-8.

Buitrago-Garcia, D., Egli-Gany, D., Counotte, M.J., Hossmann, S., Imeri, H., Ipekci, A. M., Salanti, G., Low, N., Ford, N., 2020. Occurrence and transmission potential of asymptomatic and presymptomatic SARS-CoV-2 infections: a living systematic review and meta-analysis e1003346 PLoS Med. 17 (9). https://doi.org/10.1371/ journal.pmed.1003346.

Bullard, J., Dust, K., Funk, D., Strong, J.E., Alexander, D., Garnett, L., Boodman, C., Bello, A., Hedley, A., Schiffman, Z., et al., 2020. Predicting infectious severe acute respiratory syndrome coronavirus 2 from diagnostic samples. Clin. Infect. Dis. 71, 2663-2666. https://doi.org/10.1093/cid/ciaa638.
Byambasuren, O., Cardona, M., Bell, K., Clark, J., McLaws, M.-L., Glasziou, P., 2020. Estimating the extent of asymptomatic COVID-19 and its potential for community transmission: systematic review and meta-analysis. JAMMI 5, 223-234. https://doi.org/10.3138/jammi-2020-0030.

Campbell, F., Archer, B., Laurenson-Schafer, H., Jinnai, Y., Konings, F., Batra, N., Pavlin, B., Vandemaele, K., Van Kerkhove, M.D., Jombart, T., et al., 2021. Increased transmissibility and global spread of SARS-CoV-2 variants of concern as at June 2021. Eurosurveillance 26, 2100509. https://doi.org/10.2807/15607917.ES.2021.26.24.2100509.

Chen, T., Dai, Z., Mo, P., Li, X., Ma, Z., Song, S., Chen, X., Luo, M., Liang, K., Gao, S., et al., 2020. Clinical characteristics and outcomes of older patients with coronavirus disease 2019 (COVID-19) in Wuhan, China: a single-centered, retrospective study. J. Gerontol. A Biolo. Sci. Med. Sci. 75, 1788-1795. https://doi.org/10.1093/ gerona/glaa089.

Clohisey, S., Baillie, J.K., 2019. Host susceptibility to severe influenza A virus infection. Crit. Care 23, 303. https://doi.org/10.1186/s13054-019-2566-7.

Danon, L., Read, J.M., House, T.A., Vernon, M.C., Keeling, M.J., 2013. Social encounter networks: characterizing Great Britain. Proc. R. Soc. Lond. B Biol. Sci. 280, 20131037. https://doi.org/10.1098/rspb.2013.1037.

Davies, N.G., Kucharski, A.J., Eggo, R.M., Gimma, A., Edmunds, W.J., Jombart, T., O'Reilly, K., Endo, A., Hellewell, J., Nightingale, E.S., Quilty, B.J., Jarvis, C.I., Russell, T.W., Klepac, P., Bosse, N.I., Funk, S., Abbott, S., Medley, G.F., Gibbs, H., Pearson, C.A.B., Flasche, S., Jit, M., Clifford, S., Prem, K., Diamond, C., Emery, J., Deol, A.K., Procter, S.R., van Zandvoort, K., Sun, Y.F., Munday, J.D., Rosello, A., Auzenbergs, M., Knight, G., Houben, R.M.G.J., Liu, Y., 2020a. Effects of nonpharmaceutical interventions on COVID-19 cases, deaths, and demand for hospital services in the UK: a modelling study. Lancet Publ. Health 5 (7), e375e385. https://doi.org/10.1016/S2468-2667(20)30133-X.

Davies, N.G., Klepac, P., Liu, Y., Prem, K., Jit, M., Pearson, C.A.B., Quilty, B.J., Kucharski, A.J., Gibbs, H., Clifford, S., et al., 2020b. Age-dependent effects in the transmission and control of COVID-19 epidemics. Nat. Med. 26, 1205-1211. https://doi.org/10.1038/s41591-020-0962-9.

Dong, Y., Mo, X., Hu, Y., Qi, X., Jiang, F., Jiang, Z., Tong, S., 2020. Epidemiology of COVID-19 Among Children in China. Pediatrics 145,. https://doi.org/10.1542/ peds.2020-0702) e20200702.

Donohue, J.M., Miller, E., 2020. COVID-19 and school closures. JAMA 324, 845-847. https://doi.org/10.1001/jama.2020.13092.

Ferretti, L., Wymant, C., Kendall, M., Zhao, L., Nurtay, A., Abeler-Dorner, L., Parker, M., Bonsall, D., Fraser, C., 2020. Quantifying SARS-CoV-2 transmission suggests epidemic control with digital contact tracing. Science 368, eabb6936. https:// doi.org/10.1126/science.abb6936.

Flaxman, S., Mishra, S., Gandy, A., Unwin, H.J.T., Mellan, T.A., Coupland, H., Whittaker, C., Zhu, H., Berah, T., Eaton, J.W., et al., 2020. Estimating the effects of non-pharmaceutical interventions on COVID-19 in Europe. Nature 584, 257261. https://doi.org/10.1038/s41586-020-2405-7.

Goldstein, E., Lipsitch, M., Cevik, M., 2020. On the effect of age on the transmission of SARS-CoV-2 in households, schools and the community. J. Infect. Dis. 223, 362-369. https://doi.org/10.1093/infdis/jiaa691.

Greer, A.L., Tuite, A., Fisman, D.N., 2010. Age, influenza pandemics and disease dynamics. Epidemiol. Infect. 138, 1542-1549. https://doi.org/10.1017/ S0950268810000579.

Han, M.S., Choi, E.H., Chang, S.H., Jin, B.-L., Lee, E.J., Kim, B.N., Kim, M.K., Doo, K., Seo, J.-H., Kim, Y.-J., Kim, Y.J., Park, J.Y., Suh, S.B., Lee, H., Cho, E.Y., Kim, D.H., Kim, J.M., Kim, H.Y., Park, S.E., Lee, J.K., Jo, D.S., Cho, S.-M., Choi, J.H., Jo, K.J., Choe, Y.J., Kim, K.H., Kim, J.-H., 2021. Clinical characteristics and viral RNA detection in children with coronavirus disease 2019 in the Republic of Korea. JAMA Pediatrics 175 (1), 73. https://doi.org/10.1001/jamapediatrics. 2020.3988.

Hart, W.S., Maini, P.K., Thompson, R.N., 2021. High infectiousness immediately before COVID-19 symptom onset highlights the importance of continued contact tracing. Elife 10. https://doi.org/10.7554/eLife.65534.

Hatchett, R.J., Mecher, C.E., Lipsitch, M., 2007. Public health interventions and epidemic intensity during the 1918 influenza pandemic. Proc. Natl. Acad. Sci. U. S. A. 104, 7582. https://doi.org/10.1073/pnas.0610941104.

Hellewell, J., Abbott, S., Gimma, A., Bosse, N.I., Jarvis, C.I., Russell, T.W., Munday, J.D., Kucharski, A.J., Edmunds, W.J., Sun, F., et al., 2020. Feasibility of controlling COVID-19 outbreaks by isolation of cases and contacts. Lancet Glob. Health 8 , e488-e496. https://doi.org/10.1016/S2214-109X(20)30074-7.

Huang, Q.S., Wood, T., Jelley, L., Jennings, T., Jefferies, S., Daniells, K., Nesdale, A., Dowell, T., Turner, N., Campbell-Stokes, P., et al., 2021. Impact of the COVID-19 nonpharmaceutical interventions on influenza and other respiratory viral infections in New Zealand. Nat. Commun. 12, 1001. https://doi.org/10.1038/ s41467-021-21157-9.

Ibuka, Y., Ohkusa, Y., Sugawara, T., Chapman, G.B., Yamin, D., Atkins, K.E., Taniguchi, K., Okabe, N., Galvani, A.P., 2016. Social contacts, vaccination decisions and influenza in Japan. J. Epidemiol. Community Health 70, 162-167. https://doi. org/10.1136/jech-2015-205777.

Imai, N., Gaythorpe, K.A.M., Abbott, S., Bhatia, S., van Elsland, S., Prem, K., Liu, Y., Ferguson, N.M., 2020. Adoption and impact of non-pharmaceutical interventions for COVID-19. Wellcome Open Res. 5, 59. https://doi.org/ 10.12688/wellcomeopenres.15808.1.

Kirsch, T.D., Moseson, H., Massaquoi, M., Nyenswah, T.G., Goodermote, R. Rodriguez-Barraquer, I., Lessler, J., Cumings, D.A.T., Peters, D.H., 2017. Impact of interventions and the incidence of ebola virus disease in Liberiaimplications for future epidemics. Health Policy Plan. 32, 205-214. https:// doi.org/10.1093/heapol/czw113. 
Koh, W.C., Naing, L., Chaw, L., Rosledzana, M.A., Alikhan, M.F., Jamaludin, S.A., Amin, F., Omar, A., Shazli, A., Griffith, M., Pastore, R., Wong, J., Leekha, S., 2020. What do we know about SARS-CoV-2 transmission? A systematic review and metaanalysis of the secondary attack rate and associated risk factors e0240205 PLoS ONE 15 (10). https://doi.org/10.1371/journal.pone.0240205.

Kucharski, A.J., Kwok, K.O., Wei, V.W.I., Cowling, B.J., Read, J.M., Lessler, J., Cummings, D.A., Riley, S., 2014. The contribution of social behaviour to the transmission of influenza A in a human population. PLoS Pathog. 10,. https:// doi.org/10.1371/journal.ppat.1004206 e1004206.

Lai, C.-C., Shih, T.-P., Ko, W.-C., Tang, H.-J., Hsueh, P.-R., 2020. Severe acute respiratory syndrome coronavirus 2 (SARS-CoV-2) and coronavirus disease2019 (COVID-19): The epidemic and the challenges. Int. J. Antimicrob. Agents 55,. https://doi.org/10.1016/j.ijantimicag.2020.105924 105924.

Leclerc, Q.J., Fuller, N.M., Knight, L.E., Funk, S., Knight, G.M., 2020. What settings have been linked to SARS-CoV-2 transmission clusters? Wellcome Open Res 5, 83. https://doi.org/10.12688/wellcomeopenres.

Leung, K., Jit, M., Lau, E.H.Y., Wu, J.T., 2017. Social contact patterns relevant to the spread of respiratory infectious diseases in Hong Kong. Sci. Rep. 7 (1). https:// doi.org/10.1038/s41598-017-08241-1.

Liu, Y., Gayle, A.A., Wilder-Smith, A., Rocklov, J., 2020. The reproductive number of COVID-19 is higher compared to SARS coronavirus. J. Travel Med. 27, taaa021. https://doi.org/10.1093/jtm/taaa021.

Liu, T., Gong, D., Xiao, J., Hu, J., He, G., Rong, Z., Ma, W., 2020. Cluster infections play important roles in the rapid evolution of COVID-19 transmission: a systematic review. Int. J. Infect. Dis. 99, 374-380. https://doi.org/10.1016/j. ijid.2020.07.073.

Loades, M.E., Chatburn, E., Higson-Sweeney, N., Reynolds, S., Shafran, R., Brigden, A., Linney, C., McManus, M.N., Borwick, C., Crawley, E., 2020. Rapid systematic review: the impact of social isolation and loneliness on the mental health of children and adolescents in the context of COVID-19. J. Am. Acad. Child Adolesc. Psychiatry 59, 1218-1239.e1213. https://doi.org/10.1016/j.jaac.2020.05.009.

Lovell-Read, F.A., Funk, S., Obolski, U., Donnelly, C.A., Thompson, R.N., 2021. Interventions targeting nonsymptomatic cases can be important to prevent local outbreaks: SARS-CoV-2 as a case-study. J. R. Soc. Interface 18, 20201014. https://doi.org/10.1098/rsif.2020.1014.

Ludvigsson, J.F., 2020. Systematic review of COVID-19 in children shows milder cases and a better prognosis than adults. Acta Paediatrica (Oslo, Norway : 1992) 109, 1088-1095. https://doi.org/10.1111/apa.15270.

Madewell, Z.J., Yang, Y., Longini Jr., I.M., Halloran, M.E., Dean, N.E., 2020. Household transmission of SARS-CoV-2: a systematic review and META-ANALYSIS. JAMA Network Open 3. https://doi.org/10.1001/jamanetworkopen.2020.31756.

Mehta, N.S., Mytton, O.T., Mullins, E.W.S., Fowler, T.A., Falconer, C.L., Murphy, O.B., Langenberg, C., Jayatunga, W.J.P., Eddy, D.H., Nguyen-Van-Tam, J.S., 2020. SARSCoV-2 (COVID-19): what do we know about children? A systematic review. Clin. Infect. Dis. 71, 2469-2479. https://doi.org/10.1093/cid/ciaa556).

Moore, S., Hill, E.M., Tildesley, M.J., Dyson, L., Keeling, M.J., 2021. Vaccination and non-pharmaceutical interventions for COVID-19: a mathematical modelling study. Lancet Infect. Dis. 21, 793-802. https://doi.org/10.1016/S1473-3099(21) 00143-2.

Mossong, J., Hens, N., Jit, M., Beutels, P., Auranen, K., Mikolajczyk, R., Massari, M., Salmaso, S., Tomba, G.S., Wallinga, J., et al., 2008. Social contacts and mixing patterns relevant to the spread of infectious diseases. PLoS Med. 5,. https://doi. org/10.1371/journal.pmed.0050074 e74.

Munro, A.P., Faust, S.N., 2020. COVID-19 in children: current evidence and key questions. Curr. Opin. Infect. Dis. 33, 540-547. https://doi.org/10.1097/ QCO.0000000000000690.

Nishiura, H., Cook, A.R., Cowling, B.J., 2011. Assortativity and the probability of epidemic extinction: a case study of pandemic influenza A (H1N1-2009). Interdiscip. Perspect. Infect. Dis. 2011,. https://doi.org/10.1155/2011/194507 194507.

Norris, J.R., 1997. Markov Chains (Cambridge Series in Statistical and Probabilistic Mathematics). Cambridge University Press, Cambridge.

Perra, N., 2021. Non-pharmaceutical interventions during the COVID-19 pandemic: a review. Phys. Rep. 913, 1-52. https://doi.org/10.1016/j.physrep.2021.02.001.

Pijls, B.G., Jolani, S., Atherley, A., Derckx, R.T., Dijkstra, J.I.R., Franssen, G.H.L., Hendriks, S., Richters, A., Venemans-Jellema, A., Zalpuri, S., Zeegers, M.P., 2021. Demographic risk factors for COVID-19 infection, severity, ICU admission and death: a meta-analysis of 59 studies. BMJ Open 11 (1), e044640. https://doi.org/ 10.1136/bmjopen-2020-044640.

Pollock, A.M., Lancaster, J., 2020. Asymptomatic transmission of covid-19. BMJ 371,. https://doi.org/10.1136/bmj.m4851) m4851.

Prem, K., Cook, A.R., Jit, M., 2017. Projecting social contact matrices in 152 countries using contact surveys and demographic data. PLoS Comp. Biol. 13,. https://doi. org/10.1371/journal.pcbi.1005697 e1005697.

Prem, K., Liu, Y., Russell, T.W., Kucharski, A.J., Eggo, R.M., Davies, N., Flasche, S., Clifford, S., Pearson, C.A.B., Munday, J.D., et al., 2020. The effect of control strategies to reduce social mixing on outcomes of the COVID-19 epidemic in Wuhan, China: a modelling study. Lancet Public Health 5, e261-e270. https:// doi.org/10.1016/S2468-2667(20)30073-6.

Qiu, X., Nergiz, A.I., Maraolo, A.E., Bogoch, I.I., Low, N., Cevik, M., 2021. Defining the role of asymptomatic and pre-symptomatic SARS-CoV-2 transmission - a living systematic review. Clin. Microbiol. Infect. 27, 511-519. https://doi.org/10.1016/ j.cmi.2021.01.011.

Read, J.M., Edmunds, W.J., Riley, S., Lessler, J., Cummings, D.A.T., 2012. Close encounters of the infectious kind: methods to measure social mixing behaviour.
Epidemiol. Infect. 140, 2117-2130. https://doi.org/10.1017/ S0950268812000842.

Read, J.M., Lessler, J., Riley, S., Wang, S., Tan, L.J., Kwok, K.O., Guan, Y., Jiang, C.Q., Cummings, D.A.T., 2014. Social mixing patterns in rural and urban areas of southern China. Proc. R. Soc. Lond. B Biol. Sci. 281, 20140268. https://doi.org/ 10.1098/rspb.2014.0268.

Regmi, K., Lwin, C.M., 2020. Impact of non-pharmaceutical interventions for reducing transmission of COVID-19: a systematic review and meta-analysis protocol e041383 BMJ Open 10 (10). https://doi.org/10.1136/bmjopen-2020041383.

Rossman, H., Keshet, A., Shilo, S., Gavrieli, A., Bauman, T., Cohen, O., Shelly, E., Balicer, R., Geiger, B., Dor, Y., et al., 2020. A framework for identifying regional outbreak and spread of COVID-19 from one-minute population-wide surveys. Nat. Med. 26, 634-638. https://doi.org/10.1038/s41591-020-0857-9.

Sachak-Patwa, R., Byrne, H.M., Dyson, L., Thompson, R.N., 2021. The risk of SARSCoV-2 outbreaks in low prevalence settings following the removal of travel restrictions. Commun. Med. 1, 39. https://doi.org/10.1038/s43856-021-000388.

Sadique, M.Z., Adams, E.J., Edmunds, W.J., 2008. Estimating the costs of school closure for mitigating an influenza pandemic. BMC Publ. Health 8, 135. https:// doi.org/10.1186/1471-2458-8-135.

Siebach, M.K., Piedimonte, G., Ley, S.H., 2021. COVID-19 in childhood: transmission, clinical presentation, complications and risk factors. Pediatr. Pulmonol. 56 (6), 1342-1356.

Silverman, M., Sibbald, R., Stranges, S., 2020. Ethics of COVID-19-related school closures. Can. J. Public Health 111, 462-465. https://doi.org/10.17269/s41997020-00396-1.

Spinelli, M.A., Glidden, D.V., Gennatas, E.D., Bielecki, M., Beyrer, C., Rutherford, G., Chambers, H., Goosby, E., Gandhi, M., 2021. Importance of non-pharmaceutical interventions in lowering the viral inoculum to reduce susceptibility to infection by SARS-CoV-2 and potentially disease severity. Lancet Infect. Dis. 21 (9), e296-e301. https://doi.org/10.1016/S1473-3099(20)30982-8.

Thompson, R.N., 2020. Novel coronavirus outbreak in Wuhan, China, 2020: intense surveillance is vital for preventing sustained transmission in new locations. J. Clin. Med. 9, 498. https://doi.org/10.3390/jcm9020498.

Thompson, R.N., Jalava, K., Obolski, U., 2019. Sustained transmission of Ebola in new locations: more likely than previously thought. Lancet Infect. Dis. 19, 10581059. https://doi.org/10.1016/S1473-3099(19)30483-9.

Thompson, R.N., Gilligan, C.A., Cunniffe, N.J., 2020. Will an outbreak exceed available resources for control? Estimating the risk from invading pathogens using practical definitions of a severe epidemic. J. R. Soc. Interface 17 (172), 20200690. https://doi.org/10.1098/rsif.2020.0690.

Thompson, R.N., Hollingsworth, T.D., Isham, V., Arribas-Bel, D., Ashby, B., Britton, T. Challenor, P., Chappell, L.H.K., Clapham, H., Cunniffe, N.J., et al., 2020. Key questions for modelling COVID-19 exit strategies. Proc. R. Soc. Lond. B Biol. Sci. 287, 20201405. https://doi.org/10.1098/rspb.2020.1405.

Thompson, R.N., Hill, E.M., Gog, J.R., 2021. SARS-CoV-2 incidence and vaccine escape. Lancet Infect. Dis. 21, 913-914. https://doi.org/10.1016/S1473-3099 (21)00202-4.

United Nations, Department of Economic and Social Affairs, Population Division. 2019 World Population Prospects 2019, Online Edition, Rev. 1. Available at: <https://population.un.org/wpp/Download/Standard/Population/>.

Verity, R., Okell, L.C., Dorigatti, I., Winskill, P., Whittaker, C., Imai, N., CuomoDannenburg, G., Thompson, H., Walker, P.G.T., Fu, H., et al., 2020. Estimates of the severity of coronavirus disease 2019: a model-based analysis. Lancet Infect. Dis. 20, 669-677. https://doi.org/10.1016/S1473-3099(20)30243-7.

Viner, R.M., Mytton, O.T., Bonell, C., Melendez-Torres, G.J., Ward, J., Hudson, L., Waddington, C., Thomas, J., Russell, S., van der Klis, F., Koirala, A., Ladhani, S., Panovska-Griffiths, J., Davies, N.G., Booy, R., Eggo, R.M., 2021. Susceptibility to SARS-CoV-2 infection among children and adolescents compared with adults: a systematic review and meta-analysis. JAMA Pediatrics 175 (2), 143. https://doi. org/10.1001/jamapediatrics.2020.4573.

Wallinga, J., Teunis, P., Kretzschmar, M., 2006. Using data on social contacts to estimate age-specific transmission parameters for respiratory-spread infectious agents. Am. J. Epidemiol. 164, 936-944. https://doi.org/10.1093/aje/kwj317.

Wei, W.E., Li, Z., Chiew, C.J., Yong, S.E., Toh, M.P., Lee, V.J., 2020. Presymptomatic transmission of SARS-CoV-2 - Singapore, January 23-March 16, 2020. MMWR Morb. Mortal. Wkly Rep. 69 (14), 411-415.

Wölfel, R., Corman, V.M., Guggemos, W., Seilmaier, M., Zange, S., Müller, M.A. Niemeyer, D., Jones, T.C., Vollmar, P., Rothe, C., Hoelscher, M., Bleicker, T., Brünink, S., Schneider, J., Ehmann, R., Zwirglmaier, K., Drosten, C., Wendtner, C., 2020. Virological assessment of hospitalized patients with COVID-2019. Nature 581 (7809), 465-469. https://doi.org/10.1038/s41586-020-2196-X.

Zhai, P., Ding, Y., Wu, X., Long, J., Zhong, Y., Li, Y., 2020. The epidemiology, diagnosis and treatment of COVID-19. Int. J. Antimicrob. Agents 55, 105955. https://doi. org/10.1016/j.ijantimicag.2020.105955.

Zhang, J., Litvinova, M., Liang, Y., Wang, Y., Wang, W., Zhao, S., Wu, Q., Merler, S., Viboud, C., Vespignani, A., et al., 2020. Changes in contact patterns shape the dynamics of the COVID-19 outbreak in China. Science 368, 1481-1486. https:// doi.org/10.1126/science.abb8001.

Zhao, S., Lin, Q., Ran, J., Musa, S.S., Yang, G., Wang, W., Lou, Y., Gao, D., Yang, L., He, D., Wang, M.H., 2020. Preliminary estimation of the basic reproduction number of novel coronavirus (2019-nCoV) in China, from 2019 to 2020: a data-driven analysis in the early phase of the outbreak. Int. J. Infect. Dis. 92, 214-217. https://doi.org/10.1016/j.ijid.2020.01.050. 